\title{
Gattungen und System der Kritik beim jungen Friedrich Schlegel
}

\begin{abstract}
Dieses gründliche Verstehen nun, welches, wenn es in bestimmten Worten ausgedrückt wird, Charakterisieren heißt, ist das eigentliche Geschäft und Wesen der Kritik.

Friedrich Schlegel

Eigentlicher Gegenstand der Kritik sind nur Werke und Systeme von Werken, nicht Menschen.

Friedrich Schlegel

Die Kritik muß also auch eine eigne Wissenschaft seyn!

Friedrich Schlegel
\end{abstract}

\section{Einführung}

Wer sich der Aufgabe stellt, Schlegels fragmentarische Äußerungen zur Kritik systematisch oder im systematischen Zusammenhang zu erfassen, läuft eine doppelte hermeneutische Gefahr. Die erste bestünde in dem zum Scheitern verurteilten Versuch, aus der Vielfalt und Fülle einzelner Sätze eine vollständige Theorie derselben zu konstruieren; die zweite wäre, die Schlüssigkeit des Kritikbegriffs vorwegzunehmen, als hätte Schlegel selbst seine zerstreuten und oft widersprüchlich erscheinenden Urteile unter einem Begriff einfach subsumieren wollen, eine bunte Mannigfaltigkeit in Buchstaben zu fixieren getrachtet, und nicht versucht, wie er oft betont, gebundenen Geist »fließend «bzw. »frei« zu machen. ${ }^{1}$ Meine Bemerkungen verstehe ich als ein vorläufiges Bemühen, zwischen der hier skizzierten Skylla und Charybdis der Theoribildung hin-

I Kritische Friedrich-Schlegel-Ausgabe. Hg. von Ernst Behler. Paderborn/München/Wien 1958 ff., im Folgenden zitiert als KFSA. Hier: KFSA I8, S. 344: "Ohne Buchstabe kein Geist; der Buchst[abe] nur dadurch zu überwinden, daß er fließsend gemacht wird." 
100 durchzusteuern und eine neu konzipierte Systematik in Schlegels aphoristischer Verfahrensweise zur Kritik vorzuschlagen, welche die hermeneutische Katastrophe vermeidet. Denn es liegt auf der Hand: Schlegel wollte es hinsichtlich der Kritik weder bei den sowohl historisch als auch systematisch schon herausgearbeiteten individuellen Gattungen oder Formen bewenden lassen, noch voreilig diese bloß unter einem a- oder überhistorischen Begriff subsumiert wissen, bzw. ihnen einfach unter der Ägide einer philosophischen >Idee im Sinne Hegels - als das zu-sich-selbergekommene Wissen - zu huldigen. Dies widerspricht ganz und gar der Schlegelschen Denk- und Verfahrensweise.

Schon die ältere Schlegel-Forschung hat die zentrale Stellung des frühromantischen Kritikbegriffs in den Blick gerückt. ${ }^{2}$ Sie war aber hauptsächlich auf die Literaturkritik und auf ihren Bezug zu Ästhetik und Hermeneutik gerichtet. ${ }^{3}$ Die neuere FriedrichSchlegel-Forschung wird, obwohl sie den Kritikbegriff Friedrich Schlegels viel umfassender begreift, ${ }^{4}$ seit Jahren von einer gewissen Schizophrenie heimgesucht. Es gibt zahlreiche Wissenschaftler, die einen systematischen Anspruch Friedrich Schlegels grundsätzlich bezweifeln und seine Hinweise zu einem System bzw. zur Systematik

2 Heinrich Henel: "Friedrich Schlegel und die Grundlagen der modernen literarischen Kritik«. In: Germanic Review 20 (1945), S. 8I-93; René Wellek: A History of Modern Criticism. Vol. 2. New Haven 1955; Klaus Briegleb: Ästhetische Sittlichkeit. Versuch über Friedrich Schlegels Systementwurf zur Begründung des Dichtungskritik. Tübingen 1962; Hans Eichner: „Friedrich Schlegels Theorie der Literaturkritik «. In: Zeitschrift für deutsche Philologie 88 (1969), S. 2-19; Heinz-Dieter Weber: Friedrich Schlegels Transzendentalpoesie: Untersuchungen zum Funktionswandel der Literaturkritik im I8. Jahrhundert. München 1973; Kurt Röttgers: Kritik und Praxis. Zur Geschichte des Kritikbegriffs von Kant bis Marx. Berlin 1975, S. II8-137; Hans Dierkes: Literaturgeschichte als Kritik. Untersuchungen zur Theorie und Praxis von Friedrich Schlegels Literaturgeschichtsschreibung. Tübingen I980.

3 Willy Michel: Ästhetische Hermeneutik und frühromantische Kritik. Göttingen 1982; Ernst Behler: "Von der romantischen Kunstkritik zur modernen Hermeneutik«. In: Silvio Vietta/Dirk Kemper (Hg.): Asthetische Moderne in Europa: Grundzüge und Problemzusammenhänge. München 1998. S. I26-150.

4 Jure Zovko: "Zur Aktualität von Schlegels Kritikkonzeption«. In: Bärbel Frischmann/Elizabeth Millán-Zaibert (Hg.): Das neue Licht der Frühromantik. Innovation und Aktualität frühromantischer Philosophie. Paderborn/München/Wien/Zürich 2009, S. 7I-79. 
mit großer Skepsis behandeln: Hermann Patsch, Willy Michel, Manfred Frank, und Karl-Heinz Bohrer dürften zu diesen Kritikern des Systembegriffs bei Schlegel gezählt werden. ${ }^{5}$ Andererseits gibt es auch andere Versuche - die in der jüngsten Forschung sogar zunehmen -, Schlegels Äußerungen zum System und zur Systematik ernst zu nehmen, und ihm eine echte - wenn auch völlig heterodoxe und radikal offene - Systemvorstellung zuzusprechen. ${ }^{6}$ Der erste in dieser Reihe war vielleicht Walter Benjamin, der in seiner Dissertation Der Begriff der Kunstkritik in der deutschen Romantik einen erstaunlichen Teil - mit dem Titel system und Begriff - genau diesem Thema widmete. Dort lesen wir:

Erweislich aber gegenüber allen Anzweiflungen ist, daß ihr [Schlegels und Novalis' - RL] Denken durch systematische Tendenzen und Zusammenhänge, die allerdings in ihnen selbst nur teilweise zur Klarheit und Reife gekommen sind, bestimmt wurde; oder, um es in der exaktesten und unanfechtbarsten Form auszudrücken: daß Ihr Denken sich auf systematische Gedankengänge beziehen läßt, da es in der Tat in ein richtig gewählte Koordinatensystem sich eintragen läßt, gleichviel, ob die Romantiker selbst dies System vollständig angegeben haben oder nicht. ${ }^{7}$

Außerdem heißt es bei Benjamin:

Was insbesondere die intellektuelle Anschauung betrifft, so ist Schlegels Denkweise im Gegensatz zu derjenigen vieler Mystiker ausgezeichnet durch Indifferenz gegen Anschaulichkeit; er beruft sich nicht auf intellektuelle

5 Hermann Patsch: „Friedrich Schlegels Philosophie der Philologie und Schleiermachers frühe Entwürfe zur Hermeneutik«. In: Zeitschrift für Theologie und Kirche 63 (1966), S. 444-465; Manfred Frank: Unendliche Annäherung. Die Anfänge der philosophischen Frühromantik. Frankfurt a. M. 1997; KarlHeinz Bohrer: Die Kritik der Romantik. Der Verdacht der Philosophie gegen die literarische Moderne. Frankfurt a. M. 1989. Neuerdings auch ders.: Selbstdenker und Systemdenker. Über agonales Denken. München 2012.

6 Im Folgenden werden die neueren Beiträge von Erlinghagen, Bauer, Buschmeier, Mergenthaler, Messlin und Zovko herangezogen, um gewisse Punkte meiner Analyse zu unterstützen.

7 Walter Benjamin: Der Begriff der Kunstkritik in der deutschen Romantik. Frankfurt a. M. 1973 (zuerst 1920), S. 36. 
Anschauungen und entrückte Zustande. Vielmehr sucht er, um es in eine Formel zusammenzufassen, eine unanschauliche Intuition des Systems, und er findet sie in der Sprache. Die Terminologie ist die Sphäre, in welcher jenseits von Diskursivität und Anschaulichkeit sich sein Denken bewegt. Denn der Terminus, der Begriff enthielt für ihn den Keim des Systems, war im Grunde nichts anderes als ein präformiertes System selbst. ${ }^{8}$

Benjamin schreibt den jungen Romantikern ein System zu, das aber noch nicht vollständig ausgeführt sei. Die Worte »unanschauliche Intuition" geben einen deutlichen Hinweis darauf, dass hier von keiner unmittelbaren Erfassung des Systems die Rede sein kann. Der Begriff, der Terminus als Kristallisation der Sprache schlechthin war nach Benjamin der Ort, an dem sich das System zeigt: er enthielt für ihn die Totalität des Systems in nuce, musste nur im Prozess der Reflexion weiter entfaltet und mit jeder neuen Artikulation neu ausgedrückt werden. Andere Interpreten, die auch auf irgendeine Weise ein System bzw. die Systematik in bzw. von Schlegels Denkens behaupten, sind Jure Zovko, Peter Bürger, Niklas Luhmann, Bärbel Frischmann, und -in der jüngsten Forschung - Dorit Messlin, May Mergenthaler, Manuel Bauer, Matthias Buschmeier ${ }^{9}$ und vor allem Armin Erlinghagen. ${ }^{\circ}$ Diese neueren Ansätze müssten sicherlich genauer

8 Ebd., S. 47.

9 Manuel Bauer: Schlegel und Schleiermacher. Frühromantische Kunstkritik und Hermeneutik. Paderborn/München/Wien 20II, S. 34-35. Bauer argumentiert insbesondere gegen eine Stilisierung von Schlegels Notizheften Zur Philologie zum Gründungsdokument der frühromantischen Hermeneutik; diese Rolle behält er den Charakteristiken und Kritiken vor, die für ihn die wahre Stätte Schlegelscher und frühromantischer Kritik bildet.

IO Armin Erlinghagen: "Das Konzept des ,Ganzen in Friedrich Schlegels Poetik 1793-1804«. In: Athenäum 22 (2012), S. 15-63, und ders.: Das Universum der Poesie. Prolegomena zu Friedrich Schlegels Poetik. Paderborn/München/ Wien/Zürich 20I2, S. 6IO-6I4. Erlinghagen identifiziert das Konzept des ,Ganzen` als den `Zentralgedanken` Friedrich Schlegels, versteht diesen aber als poetologisches Konzept, das sich auf die symbolische Form (S. I6, S. 63) bezieht. Diese Argumentation entspricht Erlinghagens Untersuchungsgegenstand der Poetik als 'poetisches Weltsystem ' oder 'Universum der Poesie‘, der Form als 'philosophische Poetikı (S. 22). Obwohl er den Formbegriff nicht objektivierend oder substantialisierend stilisiert und ihn als operativen 

aber die These gemeinsam, dass Schlegel nicht nur einen Anspruch auf Systematik erhebt, sondern tatsächlich in seinen Schriften ein System teilweise auch verwirklicht, oder zumindest diesem System einen Umriss verleiht. Diese Systematik oder das Systematische der Kritik bleibt aber zumeist vage oder nur approximativ, und muss vom Systemdenken der Aufklärung II und von demjenigen der Systemtheorie genauer unterschieden werden. ${ }^{12}$

Was m. E. bisher noch fehlt, und was ich im Folgenden zu leisten versuche, ist nicht nur eine genaue Lektüre der systematischen Tendenz und Absicht von Schlegels kritischer Theorie und Theorie der Kritik, um diese - ganz im Sinne Schlegels - weiter zu entfalten; vielmehr wird der Anspruch erhoben, Schlegels Systembegriff selbst als ein aktuelles und heute noch sehr gut brauchbares Modell der Systembildung im Gegensatz zur rezenten Systemtheorie und ihrer Einschätzung der Frühromantik herauszuarbeiten. ${ }^{13}$ Es gibt bis jetzt keine wahrhaft systematische Annäherung an Schlegels System der Kritik. ${ }^{14}$ Schlegel liefert uns, so meine These, eine redliche und

Begriff oder als `Funktionsbegriff^ (S. 38) spezifiziert, "vermittelst dessen das Vorgestellte sowohl, sozusagen, statisiert als auch dynamisiert werden kann" (S. I8), läßt sich fragen, ob als poetologische Bestimmung dies nur - wie hier noch zu zeigen ist - ein Moment im Gesamtsystem darstellt und von daher als begrenzt richtig, aber unvollständig bleibt. Mehr zu Erlinghagens Ansatz und Argument am Schluss dieses Beitrags.

II Siehe Andreas Arndt: "Hermeneutik und Kritik im Denken der Aufklärung«. In: Manfred Beetz/Giuseppe Cacciatore (Hg.): Die Hermeneutik im Zeitalter der Aufklärung. Köln/Weimer/Berlin 2000, S. 2II-236.

I2 Schlegel ist selbst für diese Schwierigkeit verantwortlich, weil viele seiner Formulierungen - 'Selbstdenken erregen`, ’den Blick schärfen`, oder `die Urteilskraft stärken eine Rhetorik der Aufklärung zurückrufen. Vgl. Jure Zovko: Friedrich Schlegel als Philosoph. Paderborn/München/Wien/Zürich 20Io, S. 26-27.

I3 Im letzten Teil dieses Beitrags versuche ich, die Schwächen der Systemtheorie (Luhmann, Fuchs) für eine angemessene und billige Beurteilung der frühromantischen Kritik aufzuzeigen.

I4 Bärbel Frischmann hat in einem 200I veröffentlichtem Beitrag erneut das Systematische der Theorie der Kritik bei Schlegel nachzuweisen versucht. Vgl. Bärbel Frischmann: „Friedrich Schlegels frühromantische Kritikkonzeption und ihre Potenzierung zur 'Kritik der Kritik «. In: Archiv für Begriffsgeschichte 43 (200I), S. 83-III. Frischmann besteht auf dem systematischen Zusammenhang und behauptet, Schlegels Kritikkonzeptionen folge »systematischen 
104 offene, pragmatische, genetische und generative Systemtheorie, die nicht nur die möglichen Kritiken des Systems einschliesst, sondern auch für kritische Anstöße, Umbildungen, und Ergänzungen von außen eine - um Schlegel beim Wort und Begriff zu nehmen - gewisse Empfänglichkeit aufweist. ${ }^{\text {I5 }}$ Ich hoffe auch damit Karl-Heinz Bohrer (und auch Jean-François Lyotard) die These entgegenhalten zu können, dass Innovation bzw. Agonistik und System sich nicht widersprechen müssen, öffnet sich doch jedes (für Schlegel: starke) System nicht nur der Möglichkeit, sondern der Notwendigkeit einer Kritik sowohl von innen als auch von außen. Die Ergänzung bzw. die Anschliessbarkeit der Aussagen im System konstitutieren die Standhaftigkeit und Stärke des Systems. Außerdem werden wir bei Schlegel nicht nur das eine System, sondern eine Vielfalt von Systemen feststellen, die die landläufige Kritik des Systemdenkens - das System sei das eine, alle Individualität und Eigentümlichkeit Zermalmende - gerade in Frage stellt. Die folgende Rekonstruktion gliedert sich in drei Teile: zuerst geht es um die historische Genese des Kritikbegriffs beim jungen Schlegel, sodann um den Begriff der Kritik und schließlich um Schlegels System der Kritik.

2. Zur historischen Genese des Kritikbegriffs beim jungen Friedrich Schlegel

2.I Philologische Kritik: Heyne, Wolf

Schlegel kommt, wie Ernst Behler im ersten Band der KFSA gezeigt hat ${ }^{16}$ und wie neuere Arbeiten detaillierter erweisen (und z. T. auch korrigieren), auf dem (Um-)Weg des klassichen Altertums zur Kritik, und zur klassischen Antike, die ihm zuerst durch seine Begegnung mit J. J. Winckelmann, Christian Gottlob Heyne

Bahnen« (S. 85), liefert aber nirgends einen Umriss dieses Systems. »Kritik der Kritik" oder Metakritik ist m. E. nur ein Teil von Schlegels System der Kritik und unbefriedigend als Darstellung des Gesamtsystems der Kritik.

I5 KFSA I3, S. 229.

I6 Ernst Behler: "Der Begriff der Kritik in den Studien zum klassischen Altertum«. In: KFSA I8, S. CXXXIII f. Schlegels eigene Übersicht über die 
und später mit F. A. Wolfs Prolegomena ad Homerum (1795) entgegentritt. Zusammenfassend könnte man die These vertreten, dass Schlegel Heynes Kritik als zu hermeneutisch bezeichnet, und diejenige Wolfs als zu mikrologisch-kritisch. In den Heften Zur Philologie kommt Heyne, Begründer des philologischen Seminars in Göttingen, eine wichtige Bedeutung zu, obwohl sich Schlegel auch sehr kritisch über Heyne äußert:

Heyne hält die $\varphi \lambda[$ Philologie] bloß für Hermeneutik, sieht mehr auf den historischen $\mathrm{u}[\mathrm{nd}]$ scientifischen Endzweck. Andre sehen mehr

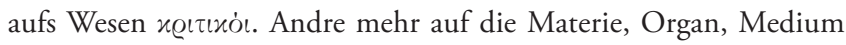

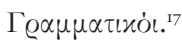

Die Heynische Interpretazion ist fast durchaus nur analytisch. Begriff einer synthetischen Interpretazion. ${ }^{18}$

Sicherlich hat Dorit Messlin recht, wenn sie behauptet, Schlegels eigener wissenschaftlicher Umgang mit dem klassichen Altertum sei eher in Abgrenzung von Heyne als unter seiner Anleitung entstanden. ${ }^{19}$ Dennoch ist die hermeneutische Leistung Heynes auch nach dem Zeugnis von Schlegels später Rezension zu Heerens Heyne-Biographie (I8I2) wichtig für das Verständnis der Genese von Schlegels Kritikbegriff. Er stimmt dort Heeren insofern zu, als Heyne - obwohl er von anderen Philologen in Holland und England übertroffen worden sei (gemeint sind sicherlich Ruhnken und Harris) - im Bereich der Textinterpretation Herausragendes geleistet habe. Schlegel schreibt in seiner Rezension, Heeren referierend: „Das Interpretieren der alten Schriftsteller sei dagegen das Fach gewesen, worin er [Heyne - RL] eigentlich geglänzt habe. $«^{20}$ Weiter schreibt er (und dies ist besonders wichtig für unsere Überlegungen zur Kritik):

Geschichte der Kritik von den Diaskeuasten bis Lessing in seinem Aufsatz Vom Wesen der Kritik (KFSA 3, S. 52-58) ist ein schönes Beispiel für seine eigene kritisch-systematisierende Methode.

I7 KFSA I6, S. 50, Nr. 177.

I8 Ebd., S. 63, Nr. 40.

I9 Dorit Messlin: Antike und Moderne. Friedrich Schlegels Philosophie, Poetik und Lebenskunst. Berlin 20II, S. 76-77.

20 KFSA 3, S. 297. 
Streng genommen läßt sich beides wohl nicht trennen. Wenn die Interpretation keinen anderen Zweck hat, als den wahren Sinn der interpretierten Schrift oder des Autors, im Einzelnen wie im Ganzen, bei allen zweifelhaften Stellen klar zu machen und gegen falsche Lesarten und falsche Erklärungen in Schutz zu nehmen und wiederherzustellen, so fällt ihr Begriff mit dem der Kritik zusammen. ${ }^{21}$

Schlegel bemängelt in dieser (späten) Rezension Heynes fehlenden kritischen Sinn und kritisiert dessen Vorliebe für die Interpretierkunst, die zum Nachteil einer strengeren, wissenschaftlichen Kritik ausschlage. Das grosse Verdienst sei Heynes sInterpretiermethoder, wie Schlegel es ausdrückt; aber die kritische Schwäche Heynes wird durchgehend betont: „Der eigentliche Vorzug der holländischen Philologen ist die höhere Kritik und die darauf gründende streng-ere Methode. ${ }^{22} \mathrm{Er}$ hat aber Heyne auch sehr geschätzt: »Heyne verband Eng.[ländische], Franz[ösische] und Deutsche Kritik und Räsonn. [ement] und Geschichte der Menschheit mit $\varphi \lambda / 2$ - formeller $\varphi \lambda$ [Philologie]. Die er aber nur sehr unvollkommen besitzt. ${ }^{23}$ Die entscheidende Stelle, an der Schlegel Heynes philosophischen Geist lobt und doch zugleich seine Mängel im kritischen Bereich (besonders der Logik und der Grammatik) bedauert, ist folgende: »Selbst Heyne durch Vereinigung $\mathrm{u}$ [nd] Verbreitung von $\varphi \sigma[$ Philosophie - RL] der Historie u[nd] der Kunst nützlich geworden. Logik u[nd] Gramm.[atik] weniger. ${ }^{24}$ Es ist kein Zufall, dass im Heft Zur Philologie I das Fragment zur Unzertrennlichkeit von Hermeneutik und Kritik direkt nach dieser Beurteilung von Heynes Art der Interpretation erscheint. Die Heynische Schule hat nach Schlegel die grammatische und kritische Philologie vernachlässigt. ${ }^{25}$

Friedrich August Wolf nimmt sowohl in den Notizheften Zur Philologie als auch in der späteren Rezension der HeyneBiographie Heerens eine weitaus höhere Stellung im Pantheon der Altertumswissenschaftler ein und erhält insbesondere den

2 I Ebd.

22 Ebd., S. 298.

23 KFSA I6, S. 43, Nr. I04.

24 Ebd., S. 55, Nr. 232.

25 Ebd., S. 53, Nr. 2II. 
kritischen Vorzug. Denis Thouard hat neuerdings die These plausibel gemacht, in Bezug auf den Kritikbegriff habe Wolf in der Philologie geleistet, was Kant in der Philosophie geleistet habe. ${ }^{26}$ In seinen Notizheften führt Schlegel Wolf als Vorbild an: „Wolfs Proleg[omena] einzig in ihrer Art durch den historischen Geist. ${ }^{27}$ Deutlicher noch heißt es: "Wolf und Winckelmann sind meine Stützen «. ${ }^{28}$ Außerdem hält er fest: »Bewunderungswürdig an Wolf, wie er seine Gränzen kennt «. ${ }^{29}$ Wolf hat nach Schlegel »heynische Universalität [...] mit der strengsten $\varphi \lambda[$ philologischen] Form der Holländer « verbunden. ${ }^{\circ}{ }^{\circ}$ Die Leistung Wolfs in der philologischen Kritik ist noch im Aufsatz Literatur aus der Zeitschrift Europa (I803) von enormer Bedeutung: »In der eigentlichen Kritik ist seit Wolfs unsterblicher Prüfung der Homerischen Gedichte nichts Bedeutendes geschehen. ॥ $^{3 \mathrm{I}}$ Dennoch hat Matthias Buschmeier vor kurzem nachgewiesen, dass Schlegel auch teilweise im Gegensatz zu Wolf seine eigene Wissenschaftsmethode entwickelt und insbesondere Wolfs These vom > Werk des Zeitalters` entschieden abgelehnt hat. ${ }^{32}$ Auch nach der Lektüre von Wolfs Prolegomena ad Homerum sieht sich Schlegel nicht vor hermeneutisch-kritische Einwände gegenüber seinem Studium-Aufsatz gestellt; vielmehr kommen bei ihm die kritischen Kategorien der sinneren Einheit wie die der Ganzheit oder Totalität des Werks erneut zur Geltung. ${ }^{33}$

26 Dennis Thouard: "Der mögliche Abschluss. Schlegel, Wolf und die Kunst der Diaskeuasten«. In: Christian Benne/Ulrich Breuer (Hg.): Antike - Philologie-Romantik. Friedrich Schlegels altertumswissenschaftliche Manuskripte. Paderborn/München/Wien 20II, S. 44-46.

27 KFSA I6, S. 39, Nr. 54.

28 Ebd., S. 52 Nr. 196.

29 Ebd., S. 40, Nr. 72.

30 Ebd., S. 43, Nr. I04.

3I KFSA 3, S. II.

32 Matthias Buschmeier: Poesie und Philologie in der Goethe-Zeit. Studien zum Verhältnis der Literatur mit ihrer Wissenschaft. Tübingen 2008, S. I2I-I23, hier: S. I20: »Behlers Einschätzung von F. A. Wolfs Einfluss auf Schlegel kann nun ein erstes Mal hinterfragt werden. Das Bewusstsein einer historischen Durchführung der Literatur- und Textkritik hat dieser keineswegs jenem zu verdanken."

33 Ebd., S. I25. Zu dem entscheidenden Schnitt F. A. Wolfs siehe Glenn Most: »Homer between Poets and Philologists«. In: David E. Wellbery (Hg.): 
Wolfs Dekonstruktion der auktorialen Einheit des Homerischen Epos' verstärke nur die innere Einheit des Werks von einem hermeneutischen Standpunkt; trotz der zersplitterten Autorschaft zeige sich mehr als zuvor das homerische Epos als einheitlicher Gegenstand der Kritik und Interpretation - allerdings endlich befreit von der Frage der empirischen Autorschaft.

\subsection{Historische Kritik: Winckelmann, Herder}

Es sind vor allem Winckelmann und Herder, denen nach Schlegel das Zeitalter die grosse Erweckung des historischen Sinns und des Sinns für historische Kritik verdankt. In den Heften Zur Philologie werden beide als bahnbrechend im Bereich der historischen Kritik angesehen: "Künftig: Methodenlehre der Philologie [der Alterthumskunde oder der philologischen Kunst?]. Andeutungen auf Winckelmann «34; »Das wichtigste Stück zu einer Philosophie der Philologie ist also eine Theorie der historischen Kritik. - Winckelmanns Historismus. ${ }^{35}$ Winckelmann hat nach Schlegel als erster die Einsicht in die rabsolute Verschiedenheit historischer Kulturen geliefert. Schlegel schreibt in den Athenaeums-Fragmenten:

Der systematische Winckelmann, der die Alten gleichsam wie Einen Autor las, alles im ganzen sah, und seine gesamte Kraft auf die Griechen konzentrierte, legte durch die Wahrnehmung der absoluten Verschiedenheit des Antiken und des Modernen den ersten Grund zu einer materialen Altertumslehre. ${ }^{36}$

In den Heften Zur Philologie steht genau diese Anerkennung von Winckelmann als Pionier auf dem Gebiet der materialen Altertumskunde im Zentrum: Schlegel rühmt »jene Ansicht von

A New History of German Literature. Cambridge/Harvard 2004, S. 503-505, sowie Anthony Grafton: »Prolegomena to Friedrich August Wolf «. In: Journal of the Warburg and Courtauld Institutes 44 (I98I), S. IOI-I29.

34 KFSA I6, S. 35 , Nr. 7.

35 Ebd., S. 36, Nr. 9.

36 KFSA 2, S. 188. 
Winckelmann hatte "gar keinen Witz, und fühlte doch die absolute Versch.[iedenheit] des Antiken und Modernen. ${ }^{38}$ Winckelmann habe - vor Herder - den echten Historismus sentdeckt $\_$, indem er die Frage nach den Bedingungen historisch-interpretatorischen Fragens und der darauf basierenden Disziplinen gestellt habe. Winckelmanns 'Einsicht habe die universitären Einheitsbestrebungen mit dem Anspruch allgemeiner Wert- und Kunsturteile aufklärerischer Prägung gestürzt: es gebe keine allgemeine, a- oder überhistorischen Maßstäbe mehr. Die Kriterien des Kunsturteils müssen aus der Materie selbst, d. h. aus dem Werk oder dem system der Werke heraus generiert werden. Schlegel lobt Winckelmann auch für seine Behandlungsart der Altertumskunde, besonders für seine `materiale`Altertumslehre und die Verabschiedung einer bloß santiquarischen Art des Studiums: "eine materiale Altertumslehre ein Beweis, wie viel man für die materiale Altertumskunde thun kann auch ohne Conjekturen zu machen und Autoren zu edieren, oder fortlaufend zu interpretieren. «" ${ }^{39}$ Winckelmanns Vertiefung in die materiale Altertumskunde, in die Materie der Antike selbst war für Schlegel ein entscheidender Schritt, denn sie ermöglichte gerade die wissenschaftliche Erforschung und Kritik der klassischen Antike vom Standpunkt der rabsoluten Verschiedenheit‘ aus.

Für Schlegel hat Herder diese rabsolute Verschiedenheit‘ historisch-kritisch weiterentwickelt und sie auf die Geschichte der Literatur und Dichtung angewandt. In der frühen Rezension zu Herders Humanitätsbriefen, erschienen 1796 in der Zeitschrift Deutschland, wird Schlegels Würdigung von Herders früheren historisch-kritischen Schriften gegenüber den Briefen deutlich.

KFSA i6, S. 47, Nr. I53. Auf der Tagung Philosophie und Philologie II in Dubrovnik hat Maren Jäger (Mainz) im Jahr 2013 in ihrem Vortrag " Mein Meister . Winckelmanns Unterscheidung von Antike und Moderne gelesen mit Friedrich Schlegel« dieses Thema der nach Schlegel durch Winckelmann zur Einsicht gekommenen sabsoluten Verschiedenheit`äußerst präzise referiert. Ich stütze mich auf ihre Argumentation.

39 Ebd., S. 42, Nr. 93. 
Wenn man sich indessen das Ganze in Gedanken mehr zusammendrängt, so darf man sich dabei immer noch an die besten kritischen Schriften des Herausgebers erinnern; d. h. an Schriften, welche den geistvollsten und zartesten Ausdruck mit der reichsten Fülle von Gedanken und Gedankenkeimen vereinigen. ${ }^{40}$

Am Ende der Rezension erläutert Schlegel Herders Kritikbegriff in den Humanitätsbriefen an einzelnen Beispielen; es handelt sich um eine ganze Reihe von Zitaten aus Herders Text: "Mangel an Kritik sollte die Krankheit nicht sein, an der der Deutsche litte«; "Die Reformation [...] war eine laut und scharf gesagte Kritik«; »Kritischer Geist der deutschen Philosophie«; »Auch die Kritik ist ohne Genius nichts. «" Und dann greift Schlegel zum Hammer:

Das Resultat leugnet, dass die Poesie verschiedener Zeiten und Völker verglichen werden könne, ja sogar, dass es einen allgemeinen Maßstab der Würdigung gebe. Aber ist dieses auch erwiesen? - Wenn noch kein tadelloser Versuch, das Feld der Poesie einzuteilen vorhanden ist, muß diese Einteilung darum überhaupt unmöglich sein? - Die Methode, jede Blume der Kunst, ohne Würdigung, nur nach Ort, Zeit und Art zu betrachten, würde am Ende auf kein andres Resultat führen, als dass alles sein müßte, was es ist und war. ${ }^{42}$

Schlegel beschuldigt die neueste Publikation Herders eines unkritischen historischen Relativismus und greift dabei scharf Herders Kritikbegriff, genauer: das Fehlen eines "allgemeinen Maßstabes der Würdigung« an. Der Herder der Humanitätsbriefe ist für den jungen Schlegel gerade nicht mehr kritisch und systematisch genug. Hier wird angedeutet, dass Schlegel spätestens 1795 ein System der Kritik, oder ein kritisches System im Auge gehabt hat, welches hermeneutisch reflektierte Kategorien und Methoden der Kritik nach der Einsicht in die rabsolute Verschiedenheit ( Winckelmann), d. h. gemäß der historischen Kluft, die uns von verschiedenen Formen, Stilen, Gattungen, Zeiten und Völkern trennt, einsetzt.

40 KFSA 2, S. 47-48.

4I Ebd., S. 54.

42 Ebd. 
Wie er in den Heften Zur Philologie betont, war Herder für ihn vor allem an Kulturwissenschaft und Kulturgeschichte interessiert: "Herders Liebe für die Alten ist wohl mehr Interesse an Cultur überhaupt. « ${ }^{43}$ Das Wort `überhaupt besagt, dass Herder mehr auf das Allgemeine und Universelle achtete. Außerdem wird deutlich, dass es Schlegel um Kunst geht, nicht um Cultur. Herder hat nach Schlegel nie eine systematische Kritik aufgestellt, noch habe er versucht, eine Grundlegung der Kritik zu liefern. Es muss nach Schlegel Maßstäbe und Kriterien geben - sicherlich nicht bloß abstrakte und der Materie völlig fremde Kategorien, die einfach rangewendet ‘ werden - um ein `wahres gemäßes Kunsturteil zu fällen. Schlegel wirft Herder vor, er habe die historische Kluft zu weit getrieben und dadurch jede Möglichkeit eines objektiven Urteils abgewiesen. In den philologischen Studienheften Von der Schönheit in der Dichtkunst bemüht sich Schlegel um eine "Theorie der Beurtheilung des Schönen«. Er interessiert sich für eine "sichere objective Methode oder Regel und Norm der Anwendung der objektiven Gesetze der Ästhetik auf einzelne Fälle, oder der Beurtheilung. «"4 Genau an diesem Punkt zeigt sich deutlich, wie sich Schlegel von Herders Theorie der historischen Kulturkritik - Kritik als Kulturbegriff - abhebt und eine wissenschaftliche Theorie der Kunstkritik zu begründen versucht.

\section{3 Ästhetische, Poetische Kritik: Lessing}

Der Einfluss Lessings innerhalb der Genese von Schlegels Kritikbegriff ist kaum zu überschätzen. Das verdeutlicht besonders die Allgemeine Einleitung zur Lessing-Edition aus dem Jahr 1804, die unter dem Titel Vom Wesen der Kritik steht. »Alles was Lessing getan, gebildet, versucht und gewollt hat «, schreibt Schlegel dort, "äßt sich am füglichsten unter den Begriff der Kritik zusammenfassen; ein Begriff, der, so mannigfaltig und weitverbreitet auch die Tätig-

$43 K F S A$ I6, S. 7I, Nr. II2.

44 Ebd., S. 5. Messlin: Antike und Moderne (s. Anm. 19), S. 374, zeigt, dass Schlegel diesen Plan keineswegs aufgegeben, sondern in den Kölner Vorlesungen weiter verfolgt hat. 
112 keit seines Geistes war, dennoch vollkommen hinreichen kann zur gemeinschaftlichen Übersicht derselben. ${ }^{45}$ Dennoch ist auch in Schlegels rühmender Würdigung Lessings die Spur einer Kritik zu finden, die man nicht übersehen sollte. Es handelt sich ganz präzise um die ästhetische Beschaffenheit der Kritik bei Lessing:

\begin{abstract}
So mancher Berichtigung also auch Lessings Begriffe von der Kunst bedürfen mögen, so führte doch seine Ästhetik wenigstens auf den rechten Weg; denn die Sonderung der Gattungen führt, wenn sie gründlich vollendet wird, früher oder später zu einer historischen Konstruktion des Ganzen der Kunst und der Dichtkunst. Diese Konstruktion und Erkenntnis des Ganzen aber ist von uns als die eine und wesentlichste Grundbestimmung einer Kritik, welche ihre hohe Bestimmung wirklich erfüllen soll, aufgestellt worden. ${ }^{46}$
\end{abstract}

Es ist nicht übertrieben zu behaupten, dass Schlegel trotz seiner Bewunderung für Lessing im Aufsatz Vom Wesen der Kritik diesen als Bühne oder Bildschirm für seine eigene Programmatik benutzte, eine Art Selbstprofilierung, mit der Schlegel sein eigenes kritisches Vorhaben zu erkennen geben konnte. Das zeigt etwa die folgende Passage:

Wir haben versucht, Lessings sämmtliche literarische Versuche unter dem Begriff der Kritik zusammenzufassen, als dem einzigen, unter den sie sich ohne Zwang vereinigen, und von wo aus sie sich als ein Ganzes mit bequemer Übersicht fassen und in ihrem sukzessiven Enstehen begreifen lassen können. [...] Die Notwendigkeit der Kritik für jede Literatur war nicht schwer zu zeigen. Der Begriff der Kritik aber, der dabei durchgängig zum Grunde gelegt ward war der historische. Es ward nur von der Kritik gesprochen, die bis jetzt gewesen ist, und wie sie es gewesen ist. Aber kann es nicht noch eine ganz andre geben? Kann die als Tatsache aus der Geschichte bekannte Kunst und Wissenschaft dieses Namens nicht eine ganz neue Wendung oder vielmehr totale Umkehrung erleben? Dies ist nicht nur möglich, sondern auch wahrscheinlich [...].47

45 KFSA $_{3}$, S. 5I-52.

46 Ebd., S. 58. Vgl. auch Erlinghagen: „Das Konzept des 'Ganzen ' in Friedrich Schlegels Poetik« (s. Anm. IO), S. 24.

47 KFSA 2, S. 8I. 
Schlegel setzt sich von Lessings Art und Weise, historische und ästhetische Kritik zu betreiben, ab, indem er eine "ganz neue Wendung", sogar eine "totale Umkehrung" ankündigt, die die Kritik als Kunst und Wissenschaft wieder im Sinne ihres Ursprungs erreichen könnte. Trotz seines erstaunlichen Scharfsinns bleibt Lessing nach Schlegel in einer Ästhetik oder einer ästhetischen Kritik befangen, die die Historizität des ästhetischen Diskurses selbst nie hinterfragt.

Es gibt aber einen anderen Aspekt der Lessingschen Kritik, den Schlegel in seinen Lessing-Aufsätzen besonders schätzt: Lessings agonales Denken, seine Bereitschaft, sich polemisch in heftige theologisch-politische wie philologische Streitigkeiten einzulassen, ja sie sogar selbst zu provozieren. Schlegel preist die explosive Gewalt von Lessings kritischem Geist. Lessings unermüdliche Kritik an der institutionalisierten Religion wie sein putativer Spinozismus zogen den jungen Schlegel an. Lessing ist für ihn als Kritiker ein begriffsprägendes Vorbild: »man sagt oft nur: ein Lessing, um einen vollendeten poetischen Kritiker zu bezeichnen. $\aleph^{48}$ Lessing hat Poesie und Kritik derart miteinander verbunden, dass sie nur durch und miteinander verstanden werden konnten: "Seine Poesie verstand er durch seine Kritik, die ebenso alt und schwesterlich aufgewachsen war. «"9 Aber Schlegel tut auch etwas mit Lessing; er ist daran interessiert, ihn "aus der Poesie und poetischen Kritik ganz wegzuheben und hinüberzuführen in jene Sphäre, wohin selbst die Tendenz seines Geistes immer mehr zog, in die Philosophie. ¿ $^{\text {so }}$ Als Kritiker sei Lessing vielleicht nicht philosophisch, d. h. nicht systematisch genug gewesen: wie Lessing selbst in der Vorrede zum Laokoon schreibt, sind die Aufgaben des Philosophen und des Kritikers, was die Kunst angeht, völlig verschieden. Lessing hat Mendelssohn als Nonplusultra der philosophischen Ästhetik angesehen; sich selbst hatte er demgegenüber die Position des Kritikers zugewiesen, dem es um die Frage geht, wie bestimmte Gattungen und Medien geeignete Arten von Zeichen mobilisieren können, die den Zuschauer zum

48 Ebd., S. 90.

49 Ebd., S. II5.

50 Ebd., S. 397. 
114 Denken provozieren und kritische Einsichten sowie einen Sinn für eine humanitäre Gemeinschaft im Leser, bzw. Zuschauer hervorzubringen geeignet sind.

\subsection{Politische Kritik: Condorcet, Forster}

Auf dem Terrain der politischen Kritik hat sich Schlegel zwei wichtige Kategorien angeeignet. Das geschieht in seiner Auseinandersetzung mit Condorcet und Georg Forster, die ich hier nur kurz erwähnen möchte. Er nennt die Kategorien ausdrücklich in seinem Aufsatz über Georg Forster (I797):

Und welche Grundbegriffe sind es, an denen Forster so standhaft festhielt? - Die unerschütterliche Notwendigkeit der Gesetze der Natur und die unvertilgbare Vervollkommnungsfähigkeit des Menschen: die beiden Pole der höhern politischen Kritik. ${ }^{51}$

Damit schliesst Schlegel nicht nur an die Theorie des Naturrechts (wahrscheinlich in Anlehnung an Herders Aufnahme und Aneignung desselben) an, sondern - und dies ist wichtig für jede Analyse der Kritik bei Schlegel - an die damals intensiv diskutierte Idee der Perfektibilität des Menschen, wie sie in Condorcets Schrift Esquisse d'un tableau historique des progrès de l'esprit humain (1795) entworfen worden ist. In den AthenaeumsFragmenten lesen wir:

5I KFSA 2, S. 87. Es ist hier vielleicht wichtig anzumerken, dass sich zwei Grundgedanken Schlegels - die Notwendigkeit der Naturgesetze und das unendliche Streben des Menschen nach Vervollkommnung - z. T. der Aneignung der Philosophie Spinozas verdanken. Vgl. Michael Elsässer: Friedrich Schlegels Transzendentalphilosophie. Hamburg I991, und Markus Enders: »Das romantische Unendlichkeitsverständnis Freidrich Schlegels«. In: DVjs 74 (2000), S. 44-83. Vgl. auch Robert S. Leventhal: »Ein vorbildliches Beispiel: Friedrich Schlegels Spinoza-Lektüre und die Entstehung seiner Hermeneutik I795-I797«. In: Martin Bollacher/Thomas Kisser/ Manfred Walther (Hg.): Ein neuer Blick in die Welt: Spinoza in Literatur, Kunst und Ästhetik. Würzburg 2010, S. 57-72. 
Der skizzierte Gedanke einer historischen Dynamik macht dem Geiste

des Condorcet so viel Ehre, als seinem Herzen der mehr als französische Enthusiasmus für die beinahe trivial gewordene Idee der unendlichen Vervollkommnung. ${ }^{52}$

Am Prinzip der historischen Dynamik bzw. der Perfektibilität hielt Schlegel fest und machte es auch für sein Konzept der Kritik fruchtbar, indem er Kritik und Philologie als eine im Prinzip unendliche und unabschliessbare Aufgabe konzipierte. Ihre zweitrangige Stellung als bloße `Hilfswissenschaft a aufgebend wird die Philologie und insbesondere die Kritik zu einem wesentlichen Bestandteil menschlicher Selbstverwirklichung und -vervollkommnung. Dass Schlegel hier von einer ’höheren politischen Kritik redet, darf nicht befremden, da er doch gerade das gegenwärtige Zeitalter der Moderne und dessen interne Struktur so wie die politischen Verhältnissen nach der Französischen Revolution, besonders deren Gewalt, einer gründlichen Kritik unterziehen will. Philologie und Kritik sind nach Schlegel nicht nur von antiquarischem Interesse oder als Stützen der Altertumswissenschaft zu verstehen, sondern bilden vielmehr »eine notwendige Aufgabe der Menschheit. «\$3 Die Offenheit und der jeweils neu zu bestimmende Inhalt dieser Aufgabe kennzeichnet Schlegels System der Kritik.

\subsection{Philosophische Kritik: Kant und Fichte}

Zuletzt soll in diesem Abschnitt ein kurzer Blick auf die philosophische Kritik geworfen werden - sowohl im Sinne der kritischen Philosophie Kants als auch im Sinne ihrer Kritik, die vor allem durch Hamann und Herder schon vor Schlegel vollzogen wurde. ${ }^{54}$

52 KFSA 2, S. 202, Nr. 227.

$53 K F S A$ I6, S. 45, Nr. I23: »Die $\phi \lambda$ [Philologie] ist nicht bloß zu allerley Dingen nütze [...] sondern eine nothwendige Aufgabe der Menschheit«.

54 Johann Georg Hamann: »Metakritik über den Purismen der Vernunft«. In: ders.: Samtliche Werke. Hg. von Josef Nadler. Bd. 3. Wien I95I, S. 286-3I4. Der Text der Metakritik in der Fassung ihrer Mitteilung an Johann Gottfried Herder am I5. September 1784 findet sich in Johann Georg Hamann: 
Unter `Kritikı versteht Schlegel in erster Linie Kants transzendentale Kritik, die der Frage nach den Bedingungen der Möglichkeit menschlicher Erkenntnis und den Grundsätzen menschlichen Glaubens und Handelns nachgeht. 55 Weiter versteht er darunter auch Fichtes Kritik der Aporien in Kants Theorie, vor allem der Annahme eines Dings-an-sich, die rein formale Bestimmung des Ichs in der ersten Kritik, und schliesslich die strikte Trennung von theoretischer Erkenntnis und ethischem Handeln. Man ahnt die große Reichweite von Fichtes Begriff der Wechselwirkung und seines Versuchs einer Vereinigung von theoretischer und praktischer Philosophie in den Grundlagen des Naturrechts von 1796-97, die Schlegel begeistert aufnahm. In Bezug auf die hier verfolgte Fragestellung sei jedoch auf den inneren Zusammenhang der philosophischen Kritik und deren Erweiterung bzw. Historisierung durch die Philologie bei Schlegel hingewiesen. In beiden Fällen - sowohl bei Kant wie bei Fichte - sieht Schlegel eine Progression der philosophischen Kritik, die dennoch unvollendet bleibt und vor allem einer historisierenden, kritisierenden und philologischen Ausweitung bedarf. Buchstäblich interpelliert Schlegel die kritisierende Philologisierung der Philosophie durch die Unterscheidung der Morpheme des Worts >Kritikı selbst, genauer durch die Differenz des Partizips:

\begin{abstract}
Nur durch die Idee von kritischer Totalität, von einer absolut kritisirten und kritisierenden Philosophie, und durch die gesetzmäßige Fortschreitung, kunstmäßige Annäherung zu dieser unerreichbaren Idee wird der $\varphi \sigma[$ Philosoph] den Beynahmen des kritischen $\varphi \sigma$ [Philosophen] verdienen: Kant ist kein kritischer, bloß ein kritisierender $\varphi \sigma[$ Philosoph]; Fichte ein kritisierter. ${ }^{56}$
\end{abstract}

Briefwechsel. Bd. 5: 1783-I785. Hg. von Arthur Henkel. Frankfurt a. M. I965, S. 210-216. Herders Metakritik ist erst 1799 entstanden, aber das Argument, alles Denken sei immer schon Sprache, wurde - Hamann zufolge - schon vom jungen Herder aufgestellt. Siehe dazu den Aufsatz von Jürgen Trabant: »Herder and Language«. In: Hans Adler/Wulf Koepke (Hg.): A Campanion to the Works of Johann Gottfried Herder. Rochester 2009, S. II7-I4I.

55 Zovko: Friedrich Schlegel als Philosoph (s. Anm. I2), S. I5.

56 KFSA I6, S. 64, Nr. 47. 
Da der `eigentliche Sitz ‘ der Kritik die Philologie sei, ${ }^{57}$ verdiene nur eine Philosophie wirklich kritisch genannt zu werden, die durch die Philologie ihrer eigenen sprachlich-historischen Bedingungen gewahr wird.

Schlegels Anmerkungen in den Lyceums- und AthenaeumsFragmenten, den Heften Zur Philologie sowie unzählige kritische Bemerkungen zu Kant und Fichte in den Fragmenten Zur Philosophie 1796-I802 legen nahe, dass die Abgehobenheit und Abstraktheit der kritischen Philosophie Kants und des transzendentalen Idealismus Fichtes von den historischen Befunden und von der kritischen Auseinandersetzung mit der Historie bzw. Philologie beide Philosophen dazu verleitet hat, ihren eigenen historischen Standpunkt bzw. ihre eigene Historizität nicht zu hinterfragen. Insofern bleiben beide - Schlegel zufolge - in ihrer jeweiligen Kritik inkonsequent: "Kritisch heißt die Philosophie der Kantianer wohl per antiphrasin; oder es ist ein epitheton ornans. ${\text { }{ }^{8}}^{8}$ Kant und Fichte verfahren nach Schlegel nicht selbstkritisch genug, und d. h. nicht historisch-kritisch genug - bezogen auf ihre eigene transzendentale Philosophie. Das wird aus den folgenden Fragmenten ersichtlich:

Aber bei Fichte muss man, wie er selbst, ohne alle Nebenrücksicht auf das ganze sehen und auf das eine, worauf es eigentlich ankommt; nur so kann man die Identität seiner Philosophie mit der Kantischen sehen und begreifen. Auch ist kritisch wohl etwas, was man nie genug sein kann. ${ }^{59}$

K. [ant] im Grunde höchst unkritisch. ${ }^{60}$

Kants [Philosophie] ist kein System [nicht objektiv]. ${ }^{61}$

Die Ganzheit s. [eines] Systems ist gerade das Subjektivste. Kein festes, bleibendes System. ${ }^{62}$

F. [ichte] ist ein kritisierter Polemiker. Er ist nicht genug absoluter Idealist, weil er nicht genug $x[$ Kritiker] und Universalist ist [...] er

57 Ebd., S. 62, Nr. 84: »Der eigent.[liche] Sitz der Kritik ist die $\phi \lambda[$ Philologie].«

$58 K F S A$ 2, S. I72, Nr. 47.

59 Ebd., S. 213, Nr. 28I.

60 KFSA I8, S. 2I, Nr. 35.

6I Ebd., S. 22, Nr. 4I.

62 Ebd., S. 22, Nr. 45. 
ist ein halber $x$ [Kritiker], offenbar auch nicht Realist genug in jeder Bedeutung und Rücksicht. ${ }^{63}$

$x[$ Kritik $]$ der $\varphi \sigma[$ Philosophie $=\varphi \lambda[$ Philologie $] \operatorname{der} \varphi \sigma[$ Philosophie $]$ das ist eins. - Da die Philosophie so vieles ja fast alles im Himmel und auf Erden kritisiert hat; so kann sie sichs ja wohl gefallen lassen, dass man sie auch einmal kritisiere. ${ }^{64}$

Kant hatte gar nicht die ganze Transc[endentalität] kritisiert. ${ }^{65}$

Auch in der $\varphi \sigma[$ Philosophie] soll nur d[as] Classische kritisirt werden, das Transzendentale aber historisirt. ${ }^{66}$

Die Philologie übt Kritik an der Philosophie - an ihren Ursprüngen, ihrer Sprache, ihrer Geschichte - und ist damit die disziplinäre Bedingung der Möglichkeit einer wahrhaft kritischen Philosophie. Die Philologie entdeckt und erhellt die historischen Bedingungen und Voraussetzungen der Philosophie.

Schlegels Kritik versteht sich zum Teil als eine Kritik an den überzeitlichen Wahrheits- und Geltungsansprüchen der Philosophie selbst, insofern und in dem Maße als die Philosophie auf eine absolute Trennung der Philosophie von der Philologie und Historie insistiert. Seine Kritik greift aber tiefer und untergräbt das Systemdenken des philosophischen Idealismus Kants and Fichtes, indem er beide als Beispiele eines historisch unreflektierten Transzendentalismus vorführt. Das Zeitalter der Kritik (Kant) muss nach Schlegel in eine Kritik des Zeitalters als eines der überzogenen Subjektivität umgewandelt werden. Das Subjekt selbst und die auf Subjektivität gegründete Kultur, die dieses hervorbrachte, muss einer historischen Kritik unterworfen werden. Schlegel setzt Kants transzendentalem und Fichtes subjektivem Idealismus einen neuen Realismus entgegen, den er Spinoza entlehnt:

The objective dimension of romantic aesthetic theory is especially apparent in the romantics' growing fondness for Spinoza in the mid I790s. For the romantics, Spinoza represented the very antithesis of the

$63 K F S A$ I6, S. 31, Nr. I34.

64 KFSA I8, S. 40, Nr. 228.

65 Ebd., S. 62, Nr. 425.

66 Ebd., S. 92, Nr. 756. 
transcendental idealism of Kant and Fichte [...]. By the I790s Schelling,

Schlegel, Hölderlin, and Novalis had come to admire Spinoza, whose realism, they believed, should be the complement to the idealism of Kant and Fichte. ${ }^{67}$

Schlegels Realismus ist aber kein Realismus des Seienden, sondern ein Realismus des Worts, der Sprache, und der Schrift; ein Realismus, der die konstituitive Macht des Diskurses in allen Bereichen menschlichen Handelns anerkennt. Er übernimmt und verwandelt Spinozas monistischen Realismus in einen Sprachrealismus. Benjamins Lektüre zufolge entfaltet sich Schlegels Kritikbegriff ganz im Bereich der Sprache und des Wortes. In den Philosophischen Lehrjahren (I796) kommt Spinoza eine ganz besondere Bedeutung zu. Er wird von Schlegel nicht nur als grosser Mystiker bezeichnet, sondern als philosophische Alternative zu Kant und Fichte; er wird als Philosoph der Immanenz angesehen. Dass belegen die folgenden Bemerkungen: »Spinoza sogar die Identität des Idealen und Realen «; ${ }^{68} » \operatorname{Im}$ Spinoza ist das Verhältnis d[er] Theile nicht bloss abstrakt, sondern organisch; “"9 "In dem System der [Philosophie] alle die Arten verbunden wie im Spinoza. «70 Im Gespräch über die Poesie ist Spinoza gleichermaßen gerade der Philosoph, der eine Vorahnung des neuen Realismus gibt: die Interpretation seines Denkens im Gespräch folgt Schlegels Forderung eines neuen Realismus. $^{71}$

67 Frederick Beiser: The Romantic Imperative: The Concept of Early German Romanticism. Cambridge MA 2003, S. 77. Anders Arndt und Zovko, die Schlegels Transzendentalphilosophie als eine Synthese aus Idealismus und Realismus verstehen; Andreas Arndt/Jure Zovko: „Einleitung«. In: Andreas Arndt/Jure Zovko (Hg.): Friedrich Schlegel. Schriften zur kritischen Philosophie 1795-I805. Hamburg 2007, S. VII-LXIV, hier: S. XXXV: "Schlegel entfaltet seine Transzendentalphilosophie als eine Synthese der auf der substantia infinita beharrenden Spekulation Spinozas und der im Selbstbewusstsein kreisenden Reflexion Fichtes. Dabei geht es ihm in erster Linie um eine Historisierung dieser zwei gegensätzlichen, seiner Ansicht nach jedoch komplementären Denktypen, nämlich des Realismus und des Idealismus«.

$68 K F S A$ I8, S. 4I.

69 Ebd., S. 50.

70 Ebd., S. 500.

7I $\quad K F S A$ 2, S. 315-317. 
Schlegels Kritikbegriff entwickelt sich aus den fünf oben skizzierten Strömungen, entsteht aber nicht plötzlich als explosive Geburt. In den frühen Schriften zum klassischen Altertum liegen bereits die Keime seiner Kritiktheorie, besonders in seiner Behandlung der Diaskeuasten und der klassischen Kritik. Im Studium-Aufsatz finden sich Spuren des weiter gereiften Konzepts, aber der sorgfältigen Lektüre zeigen sich auch gewisse entscheidende Brüche, insofern Schlegel seinem Kritikkonzept in den Jahren I795-I797 den Feinschliff zu geben versucht. In den Heften Zur Philologie erhalten wir die ersten kohärenten Hinweise darauf, wie sich die Kritik in seinem Denken im Laufe der nächsten Jahre darstellen wird. Die entscheidenden Elemente sind, wenn auch nicht vollständig artikuliert, in den Heften Zur Philologie schon in nuce enthalten. Schlegels Begriff der Kritik erfährt dann seine Präzision wie seine philosophische Grundlegung I804 in dem Aufsatz Vom Wesen der Kritik, der wie gesagt die Edition Lessings Gedanken und Meinungen einleitet. Dort wird besonders der notwendig systematische Charakter der Kritik als Mittelglied zwischen Philosophie und Historie angesprochen:

Jetzt noch einige Worte, um zum Beschluss dieser Einleitung wenigstens anzudeuten, wie man sich den Begriff der Kritik noch genauer und wissenschaftlicher zu bestimmen habe. Man denke sich die Kritik als Mittelglied der Historie und Philosophie, das beide verbinden, in dem beide zu einem neuen Dritten vereinigt sein sollen [...]. Es versteht sich von selbst, dass hier nicht die Kompilationen der Meinungen und Systeme gemeint sein können, die man wohl so nennt. ${ }^{72}$

Der seigentliche Sitz der Kritik ist die Philologie, und darum kann die Philologie kein blosses Aggregat von Wissenschaften sein, sondern muss selber ein System bilden bzw. systematisch vorgehen. Schon im ersten Heft Zur Philologie schreibt Schlegel, die Philologie sei keine blosse Ansammlung oder Anhäufung von Wissen oder Formen. Sie muss systematisch aufbauen: „Die 
Philologie ist kein Aggregat von Wissenschaften, sondern ein Ganzes. "73 Sehen wir uns dieses System der Kritik und Schlegels Weg zu einem systematischen Anspruch näher an.

In einem ersten Schritt soll Schlegels Potenzierung oder Radikalisierung des Wolfschen Ansatzes in den Blick gerückt werden. Wolfs Prolegomena hatten dem Zeitalter schlagend vor Augen geführt, dass die homerischen Epen kein seinheitliches` Werk im traditionellen Sinne sind. Dennoch ist in der Kritik dieser Epen der Mythos der Einheit der klassischen Antike, ein geschlossenes, objektives 'System ‘ der Künste, des Mythos, der Philosophie und der Literatur, unberührt geblieben. Schlegel attackiert in seinen frühen Schriften gerade diesen unbefragten Grundsatz alterumswissenschaftlichen Denkens, indem er die Einheit und Ganzheit der klassischen Kritik und Kunst selbst in Frage stellt:

Der unglücklichste Einfall, den man je gehabt hat, und von dessen allgemeiner Herrschaft jetzt noch viele Spuren übrig sind, war es: der Griechischen Kritik und Kunsttheorie eine Auktorität beizulegen, welche im Gebiet der theoretischen Wissenschaft durchaus unstatthaft ist. ${ }^{74}$

Kurz darauf heißt es:

Die griechischen und römischen Dichter waren aber (nach Fragmenten, Nachrichten und Analogie zu urteilen) so wenig im Besitz eines vollendeten Systems objektiver ästhetischer Wissenschaften [...] daß nicht einmal der Versuch, der Entwurf, geschweige denn ein stetes Streben nach einem solchen System vorhanden war. ${ }^{75}$

Schlegels Argument erschliesst sich leicht aus seiner kritischen Ansicht der Klassizität als mobiles Konzept für die Anwendung kritischer Kategorien auf ein Werk oder System von Werken. Auktorität und ein vollendetes System >objektiver ästhetischer Wissenschaften sind eher Resultate oder Produkte unserer Erforschung einer bestimmten historischen Kultur als Attribute dieser Kultur

$74 K F S A$ I, S. 349.

75 Ebd., S. 350. 
122 selbst. Objektive Vollendung, geschlossene Systeme, Auktorialität und dergleichen sind kritische Urteile und nicht ontologisch in dieser (oder irgendeiner) Kultur verankert. Sie mögen noch so nützlich und ergiebig für ein schönes, rundes Bild der klassischen Antike sein - dennoch dürfen wir nicht vergessen (so Schlegels hermeneutischer Imperativ), dass solche Urteile eben historische, menschliche, zweckmäßige `Leistungen « und künstliche `Hervorbringungen sind, die die Kritik erst möglich machen und in der Gegenwart eine bestimmte kulturelle Funktion erfüllen: „Der $\varphi \lambda\left[\right.$ Philolog] «, schreibt Schlegel, »ist ein historisches Subjekt. « ${ }^{76}$

Das zweite entscheidende Moment ist nach der Überzeugung des jungen Friedrich Schlegel die Leistung der kritischen Philosophie Kants und Fichtes. In der Historisierung des Transzendentalen - um Jure Zovkos entscheidendem Beitrag zu folgen ${ }^{77}$ - möchte Schlegel die Möglichkeit einer selbstreflexiven Deduktion der historischen Bedingungen der Möglichkeit eines jeden kulturellen Systems aufrechterhalten:

\begin{abstract}
Aber seit durch Fichte das Fundament der kritischen Philosophie entdeckt worden ist, gibt es ein sichres Prinzip, den Kantischen Grundriß der praktischen Philosophie zu berichtigen, zu ergänzen, und auszuführen; und über die Möglichkeit eines objektiven Systems der praktischen und theoretischen ästhetischen Wissenschaften findet kein gegründeter Zweifel mehr statt. ${ }^{78}$
\end{abstract}

Die Historisierung von Kants Kritik der Urteilskraft führt nach Schlegel zu einer völligen Umarbeitung des Begriffs des `Klassischen.${ }^{79}$ Interesselosigkeit und Objektivität sind nach Schlegel keine wesentlichen, universellen, überzeitlichen Bestandteile der Schönheit, sondern durchaus historischen Ursprungs. Sie werden vom Standpunkt des Modernen aus auf die klassische Antike projiziert. Das Klassische wird losgelöst bzw. befreit von

76 KFSA i6, S. 49, Nr. I65.

77 Zovko: Friedrich Schlegel als Philosoph (s. Anm. I2), S. I5, S. 72 f.

$78 K F S A$ I, S. 357.

$79 \mathrm{Zu}$ Schlegels Historisierung von Kants Kritik der Urteilskraft vgl. Robert S. Leventhal: The Disciplines of Interpretation. Lessing, Herder, Schlegel and Hermeneutics in Germany, 1750-I800. Berlin 1994, S. 268-274. Siehe auch: Zovko: Friedrich Schlegel als Philosoph (Anm. I2), S. 9-I7. 

des klassischen Altertums. Die neue hermeneutisch-kritische Kategorie der `Klassizität` wird dann in Bewegung gesetzt als die zugrundeliegende, wesentliche Struktur einer jeden historischen Kultur, aber auch als eine Eigenschaft bestimmter vorbildlicher, herausragender und den Kern einer solchen Kultur genau treffender Werke oder Systeme von Werken etabliert: in der Moderne sind es vor allem Shakespeare, Spinoza, Kant, Goethe, und Fichte, die auf den Status (und die Bezeichnung) >moderner Klassizität`Anspruch erheben. Dies ist für die hier verfolgte Fragestellung von erheblicher Bedeutung, weil Schlegel seinen Kritikbegriff eben ans Konzept des Klassischen im Sinne von Klassizität bindet. Belegen lässt sich das durch die folgenden Zitate:

Philologie ist ein logischer Affekt, das Seitenstück der Philosophie, Enthusiasmus für chemische Erkenntnis [...]. Durch eine kunstmäBige Ausbildung jenes Sinns entsteht die Kritik, deren Stoff nur das Klassische und schlechthin Ewige sein kann, was nie ganz verstanden werden mag. ${ }^{80}$

Aber lehren soll ihn die hohe Wissenschaft echter Kritik, wie er sich selbst bilden muß in sich selbst, und vor allem soll sie ihn lehren, auch jede andre selbständige Gestalt der Poesie in ihrer klassischen Kraft und Fülle zu fassen.. $.8^{81}$

Dieses Beides, die Auswahl der klassischen Schriftsteller, welche das Ganze der griechischen Posie und Literatur in eine deutliche Ordnung stellen sollte, und zweitens die Behandlung der verschiedenen Lesarten, bleiben immerfort die Angeln der alten Kritik. ${ }^{82}$

Die gesamte $\varphi \lambda[$ Philologie] ist gewissermaßen nichts anders als Kritik. Die Kritik als Kunst kann nur an Schriften geübt werden, und zwar an klassischen. Alles ist vereinigt hier: poetische Kritik, grammat[ische], philologische, historische, philosophische. Dasselbe gilt wohl auch von Grammatik und Hermeneutik. ${ }^{83}$

80 KFSA 2, S. 24I, Nr. 404.

$8 \mathrm{I}$ Ebd., S. 284.

82 KFSA 3, S. 53.

$83 K F S A$ i6, S. 47, Nr. I54. 
Der Schritt von der Klassik - sei es im Sinne der klassischen Antike oder der Tradition einer Disiziplin der klassischen Kritik - zur >Klassizität bahnt Schlegels Weg zur zweiten Phase des Kritikbegriffs - nachlesbar in den Heften Zur Philologie - wobei die Kritik erstens (I) nicht abgekoppelt von der Hermeneutik praktiziert werden kann; (2) zweitens sich nur an klassischen Werken und nur an Werken oder Systemen von Werken verwirklichen läßt; und drittens (3) prinzipiell einer unendlichen und unabschliessbaren Ausführung fähig ist. Das hermeneutisch-interpretatorische Moment einer jeden Kritik wird deutlich, zumal die Kritik, wie Peter Bürger nachgewiesen hat, eine vorläufige Entscheidung bereits über den Wert und die Stellung des Werks im System zur Voraussetzung hat. ${ }^{84}$ Ein vorläufiges Kunsturteil, welches dem Werk den Status des Klassischen verleiht, ist die Vorbedingung der höheren Kritik. Die ganze hermeneutische Arbeit hebt erst dann an. Die genaue Beziehung zwischen niederer Kritik und Hermeneutik - also die konkrete Arbeit am Text - führt Schlegel zu den berühmten sphilologischen Antinomien ‘.

Antinomien der Philologie. Deren sind wohl sehr viele. Kritik der Philologie. - Die Deduktion muß der weitern Kritik en detail vorangehen. $<\mathrm{Zu}$ den Grundsätzen und Gesetzen>.85

Ueber den Primat der Kritik oder der Hermeneutik findet eine wahre Antinomie statt. ${ }^{86}$

Hermeneutik und Kritik sind absolut unzertrennlich dem Wesen nach, ob sie gleich in Ausübung, Darstellung getrennt werden können, und die Tendenz jeder $\varphi \lambda\left[\right.$ Philologie] auf einer Seite gewöhnlich überwiegt. ${ }^{87}$ Eigentlich müßte auch noch eine $\varphi \sigma[$ Philosophie] der Kritik vor dem $\varphi \lambda[$ philologischen] Codex vorangehen. Gewissermaßen ist beydes identisch und dieser Codex selbst $\varphi \sigma\left[\right.$ Philosophie] der Kritik. ${ }^{88}$

84 Peter Bürger: »Begriff und Grenzen der Kritik«. In: Merkur 63 (2009), S. I023-I034.

85 KFSA I6, S. 56, Nr. 239.

86 Ebd., S. 55, Nr. 236.

87 Ebd., S. 50, Nr. 178.

88 Ebd., S. 69, Nr. 8I. 
Die Antinomien der Philologie, die Schlegel in den Heften Zur Philologie aufdeckt, sind nicht zu beheben oder auszuräumen; sie sind für Schlegel konstitutiv. Die Kritik bewegt sich immer schon in den von diesen Antinomien gesteuerten hermeneutischen Spiralen. Die Hermeneutik ihrerseits geht immer mit bestimmten kritischen Kategorien ans Werk. ${ }^{89}$ Beide rekurrieren stets auf einander und müssen in ständiger Wechselwirkung stehen. Es ist Schlegels Verdienst, diese , Wechselwirkung`nicht nur als fröhlich-unverbindliche Wechselbeziehung, sondern ebensosehr als eine zutiefst problematische Verschränkung von Kritik und Hermeneutik deutlich und explizit vor Augen geführt zu haben. Dies ist nirgends klarer formuliert und zur Präzision gebracht als im Heft Zur Philologie II:

Die Antinomien über das Wesen der $\varphi \lambda$ [Philologie] über d[en] Primat, Verhältnis und Zahl der Bestandtheile müssen freyl[ich] jetzt schon mitgenommen werden. ${ }^{9 \circ}$

Bezeichnend für Schlegels Theorie der Kritik ist sein Vorhaben, die innere Verflechtung von Philosophie und Philologie, von Wissenschaft und Kunst, von diskursivem Denken und intuitiver Anschauung zunächst bewußt zu machen und dann in der praktischen Kritik selbst zu realisieren. ${ }^{91}$ Schon im Athenaeum argumentiert Schlegel für die ,Vermählung`, ’Eher oder `Hochzeit` von Philosophie und Poesie: »Alle Kunst soll Wissenschaft, und alle Wissenschaft soll Kunst werden, Poesie und Philosophie sollen vereinigt sein $«^{92}$, heißt es da. Ähnlich formuliert er es in einer Notiz: »In der Kritik ist die Hochzeit der $\varphi \lambda[$ Philologie] und $\varphi \sigma\left[\right.$ Philosophie] zur Konstitution der Wahrheit.— ${ }^{93}$ Insofern Kritik

89 Die klassische Hermeneutik seit Heidegger nannte dies die Vorstruktur des Verstehens. Vgl. Hans-Georg Gadamer: Wahrheit und Methode: Grundzüge einer philosophischen Hermeneutik. Tübingen 1960, S. 250-256.

90 KFSA I6, S. 59, Nr. 3.

9I Vgl. Benjamin: Der Begriff der Kunstkritik (s. Anm. 7), S. 42. Interessant ist Benjamins Behauptung, Schlegel habe seine Kritik jenseits von Diskursivität und Anschauung verortet.

$92 K F S A$ 2, S. I6I, Nr. II5.

$93 K F S A$ I8, S. 272, Nr. 925. 
auch als `Kunst ` oder in ihrer konkreten Iteration als `Kunstwerk angelegt ist, verursacht sie notwendig eine Potenzierung bzw. Steigerung des Kunstwerks und ist selbst ein Kunstwerk in der zweiten Potenz. Kritik muss poetisch werden oder selbst Poesie sein: "Poesie kann nur durch Poesie kritisiert werden«, schreibt Schlegel im Athenaeums-Fragment II7.94 In einer Notiz hält er fest: "Die wahre Kritik ein Autor in der 2t Potenz. «95 Die Kritik charakterisiert er in seinem Beitrag Vom Wesen der Kritik als "Mittelglied der Historie und der Philosophie, das beide verbinden. ${ }^{96}$ Im Falle der `Philologisierung` der Philosophie, die eine Bewusstseinssteigerung beider Disziplinen beinhaltet, geschieht eine Durchdringung von strenger, wissenschaftlicher, systematischer wie empirisch-mikrologischer Forschung einerseits und geistreicher Eingebung und Intuition andererseits: »In $x[$ Kritik] ist W[issenschaft] und K[unst] durchdrungen. «97 In seinen Frühschriften wendet Schlegel wiederholt gegen die herkömmliche Altertumswissenschaft ein, es fehle ihr an "Kunstsinn $",{ }^{98}$ genau wie es nach Schlegel den Jüngeren an "gründlicher Gelehrsamkeit" fehle. ${ }^{99}$ Aber Schlegel bleibt - wie Benjamin schon früh festgestellt hat - jedem Mystizismus der Kritik und des Kunsturteils fern. In den Lyceums-Fragmente wendet er sich gegen "mystische Kunstliebhaber, welche jede Kritik für Zergliederung, und jede Zergliederung für Zerstörung des Genusses halten. «100 Zumindest in seiner Theorie der Kritik verfällt Schlegel durchaus nicht dem Mystizismus.

Besonders wichtig ist daher der begriffliche Status der $A n$ schauung bei Schlegel und seine Einbeziehung des intuitiven Moments der Anschauung in die Kritik. In dem Aufsatz Vom Wesen der Kritik wird die "poetische Anschauung" als "die erste

$94 K F S A$ 2, S. I62, Nr. II7.

$95 K F S A$ 16, S. 52, Nr. 196.

$96 K F S A_{3}$, S. 60.

$97 K F S A$ I8, S. 380, Nr. 7I2.

98 Schlegel ist in dieser Beziehung sehr kritisch der Schule Heynes gegenüber; vgl. KFSA I6, S. 4I, Nr. 46: "Heynische Schule. Er glaubt auch Geschmack zu haben u.[nd] hat doch gar keinen."

99 KFSA 3, S. 55 .

IOO KFSA 2, S. I54, Nr. 57. 
und wesentlichste Bedingung der Kritik « ${ }^{\text {Ior }}$ bezeichnet. „Die erste Bedingung alles Verständnisses", schreibt Schlegel dort, "und also auch das Verständnis jedes Kunstwerks, ist die Anschauung des Ganzen. ${ }^{102}$ Im Athenaeum liest man: "Von einem Charakter gibt es keine andere Erkenntnis als Anschauung. ${ }^{103}$ Schlegel bricht hier entscheidend mit der philosophischen Begriffsbildung des I8. Jahrhunderts, mit Kants Formen der inneren (Zeit) und äußeren Anschauung (Raum), aber auch mit Fichtes intellektueller Anschauung, in der nichts anderes als die absolute Identität des Ichs unmittelbar mittels der Reflexion gestiftet wird. Schlegel versteht die Anschauung - anders als den Eindruck oder die Sinnesempfindung - als eine begreifende. Nur auf diese Weise lassen sich die entsprechenden Aussagen Schlegels mit Benjamins These einer > unanschaulichen Intuition` vereinbaren; denn Anschauung wird bei Schlegel gerade nicht als pures Sehen aufgefasst. Hier läßt sich eine klare, konsequent verfolgte Linie von seinen kritischen Schriften der Athenaeums-Zeit bis hin zu den Kölner-Vorlesungen (I804) ziehen. Dort liest man in der Darstellung der Logik: „Im Gegensatz der von vielen behaupteten rein sinnlichen Vorstellung oder Anschauung kann man den Begriff erklären als eine Anschauung, die zugleich Ahnung und Erinnerung ist. "104 Diese Veranschaulichung des Begriffs, die zugleich eine Historisierung bzw. Temporalisierung der Anschauung selbst mit sich bringt, verwischt eben die kategoriale Unterscheidung zwischen Begriff und Anschauung. Später, in den Vorlesungen zur Transzendentalphilosophie, schreibt er:

Das Verhältnis der Anschauung zu den Begriffen ist dadurch erklärt worden, daß man zu beweisen suchte, wie alle Vorstellungen zu Begriffen erhoben und ausgebildet werden können, daß also Anschauungen von Begriffen nicht wesentlich verschieden, sondern nur unreife, unentwickelte, ungebildete Begriffe sind. ${ }^{\text {105 }}$

IOI $K F S A_{3}$, S. 55 .

IO2 Ebd., S. 56.

I03 KFSA 2, S. 223.

IO4 KFSA I3, S. $23 \mathrm{I}$.

IOS Ebd., S. 287. 
Die Einfügung des Zeitlichen und damit auch des historischen Moments verleiht der Anschauung eine Tiefe oder Bandbreite, die sie bisher nicht besaß: auf das Vergangene wie auf das Zukünftige bezogen erhält die Anschauung eine Schlüsselstellung in der Theorie der Kritik. Denn die Kritik beginnt mit der Anschauung des Ganzen und ist ohne sie gar nicht möglich. Diese Anschauung bildet die erste Stufe oder Ebene jeder Kritik. Der Sinn für das Werk enthält nach Schlegel bereits ein intuitives Verständnis. Solche Argumente sind nicht nur von historischem Interesse, denn sie erinnern an das zentrale Argument von Eckhart Försters Buch The Twenty Five Years of Philosophy: A Systematic Reconstruction, in dem Förster eben den Zwitterbegriff des intuitive understanding als Antipoden zur intellektuellen Anschaunng zu rekonstruieren versucht und dadurch Goethe und Spinoza gewissermaßen gegen Kant und Fichte ausspielt. ${ }^{106}$ Schlegel ist mit seinem tiefen und breiten Begriff von Anschauung (in Anlehnung an Spinozas scientia intuitiva) und mit seiner Kritik an Fichtes intellektueller Anschauung durchaus in diesen von Förster rekonstruierten Diskurs einzuordnen.

Zwei weitere Aspekte müssen noch berücksichtigt werden, bevor das Problem des Systems bzw. der Systematik der Schlegelschen Kritik aufgeworfen werden kann: I) die Methode der immanenten Kritikı and 2) der Imperativ der Mitteilung der Kritik. Die Überzeugung, dass Schlegel in seinem Aufsatz Über Goethes Meister (1798) am deutlichsten das moderne Konzept einer ,immanenten Kritikı entfaltet habe, stammt von Benjamin, wird aber immer wieder als Grundstein des Kritikbegriffs aufgegriffen. Peter Bürger hat 2009 das Argument erneut eingebracht: Schlegel markiere in seinem Kritikbegriff »die historisch neue Situation der Moderne. ${ }^{107}$ Der Kritiker bewerte nach Schlegel das Kunstwerk

I06 Eckhard Förster: The Twenty Five Years of Philosophy: A Systematic Reconstruction. Cambridge (Harvard) 20I2, S. 249-26I. Nach Förster hat vor allem Goethe in seiner Version des Spinozismus' diese randere Art des Erkennens verwirklicht. Es liesse sich aber ebenso überzeugend nachweisen, dass Friedrich Schlegel gerade in seiner Aneignung Spinozas genau eine solche Konvergenz von Anschauung und Diskursitivität in seinem Kritikbegriff herzustellen bemüht ist.

I07 Bürger: "Begriff und Grenzen der Kritik» (s. Anm. 84), S. Io24. 
nicht nach vorgegebenen Kategorien oder Normen, sondern müsse dieses saus sich selbst heraus verstehen lernen. Denn »das Kunstwerk selbst«, so Bürger, »enthalte jene Elemente, nach denen es gelesen, kritisiert und verstanden werden will. $\aleph^{\text {108 }}$ Auch Dorit Messlin vertritt die These einer »immanenten Kritik « bei Schlegel. ${ }^{\text {Io9 }}$ Solche simmanente Kritik hat den Vorteil, dass das Werk selbst als einheitliches Ganzes, seine Form und sein Gehalt, als etwas Individuelles und Eigentümliches, einen `Sinn` enthält, und so als der fixe Gegenstand der Kritik beharrt. Schlegels Meister-Essay scheint dieses Argument zu bestätigen, heisst es doch dort:

Und was kann wohl anders entstehn als ein Gedicht, wenn ein Dichter als solcher ein Werk der Dichtkunst anschaut und darstellt? Dies liegt nicht darin, dass sie über die Grenzen des sichtbaren Werks mit Vermutungen und Behauptungen hinausgeht. ${ }^{\text {IIO }}$

Viele Formulierungen in den Fragmenten tragen ebenfalls zu diesem Ergebnis bei: »ein eigenes selbständiges Wesen für sich «"IIr; "wie das Ganze konstruiert ist « ${ }^{\mathrm{II2}}$; "die Organisation des Werks « ${ }^{\mathrm{II}}$. Auch andere Sätze Schlegels verleiten zu dieser Annahme, so etwa der folgende Satz aus Schlegels Rezension von Schillers Horen in der Zeitschrift Deutschland (I796):

Die Kritik ist weit mehr als eine bloße ästhetische Auslegungskunst, zu der sie hier herabgewürdigt wird; obgleich im edelsten Sinne des Worts, für die Kunst, den großen Sinn schöpferischer Werke rein und vollständig mit scharfer Bestimmtheit zu fassen und zu deuten. ${ }^{\mathrm{II}}{ }^{4}$

I08 Ebd., S. I033. Dierkes: Literaturgeschichte als Kritik (s. Anm. 2) hat genau dies lange zuvor schon gezeigt.

Io9 Messlin: Antike und Moderne (s. Anm. 19), S. 389: "Dass dieser Vorgang selbst eigentlich nicht historisch zu nennen ist, sondern seinerseits ein poetisches Verfahren meint, begründet sich durch die besondere Absicht frühromantischer Kritik zur immanenten Erklärung.»

IIO KFSA 2, S. I40.

III Ebd., S. I27.

II2 Ebd., S. I3I.

II3 Ebd., S. I35.

II4 Ebd., S. I4. 
Die Kritik soll Werke nicht nach einem allgemeinen Ideal beurtheilen, sondern das individuelle Ideal jedes Werks aufsuchen. ${ }^{\text {II }}$

Ohne die wohl fundierte These von der immanenten Kritik^ bei Schlegel gänzlich zu verwerfen, müsste man doch genauer zwischen verschiedenen Momenten des kritischen Prozesses einerseits, und verschiedenen Arten bzw. Ebenen der Kritik andererseits unterscheiden. Darin liegt eine entscheidende Differenz zum geläufigen Verständnis von simmanent « und simmanenter Kritik`. Wahr ist für Schlegel, dass das Werk selbst als einheitliches und eigentümliches Ganzes im Vordergrund der Kritik steht, und primär raus sich selbst heraus verstanden werden muss. Schlegel trennt die Autorintention deutlich von der Werkintention. Ebenso entscheidend ist aber bei Schlegel, dass die Kritik auch als erweiternder und ergänzender Verstehensprozess das Werk erstens im System der Werke des Autors erfassen, und zweitens das Werk ıencyklopädisch`im System der Kultur und zusammen mit anderen Werken der Gattung und des Zeitalters zu deuten suchen muss. ${ }^{116}$ Das klassische Werk wird in seinem Vorbildcharakter für das Literatur- und Schriftsystem, in dem es angesiedelt ist, auf dieses System bezogen. Die sproduktiver Kritik hält nicht vor dem einzelnen Werk inne. Ganz im Gegenteil. Sie schreitet fort und bildet das schon Gebildete weiter, sie ist eine Art Naturprodukt, eine sich organisierende, regulierende und sich selbst bildende Denkeinstellung. ${ }^{\text {II7 }}$ In Über Goethes Meister schreibt Schlegel: „Der Dichter und Künstler hingegen wird die Darstellung von neuem darstellen, das schon Gebildete noch einmal bilden wollen; er wird das Werk ergänzen, verjüngen, neu

II5 KFSA I6, S. 270, Nr. 197.

II6 Vgl. hierzu die sehr pointierte Analyse von Frischmann: „Friedrich Schlegels frühromantische Kritikkonzeption" (s. Anm. I4), S. IOO-IO3.

II7 Vgl. dazu auch die scharfsinnige Analyse von Christian Benne: »Kunst der Organisation. Zur Philologie der >Massen in Friedrich Schlegels Über Goethes Meister«. In: Ulrich Breuer/Remigius Bunia/Armin Erlinghagen (Hg.): Friedrich Schlegel und die Philologie. Paderborn u. a. 2013, S. 99-I2I. Benne gelingt es zu zeigen, dass Schlegel Kants Organismusbegriff auf den Bereich der Kunst überträgt. 
gestalten. «"18 In den Notizen heisst es: »Kritik - als d[ie] Kunst, Werke zu bilden, frei[lich] auch umzubilden, zu behandeln, zu diaskeuasieren, zu kritisieren. «I9 $^{\text {In }}$ Dieser ZZusatz ‘ der Kritik findet innerhalb des Aufsatzes Vom Wesen der Kritik im Abschnitt ,Vom kombinatorischen Geist‘ seinen prägnanten Ausdruck:

Mit der Veränderung dieses Verhältnisses aber ist auch schon die Möglichkeit und die Idee einer Kritik von ganz andrer Art gegeben. Einer Kritik, die nicht so wohl der Kommentar einer schon vorhandenen, vollendeten, verblühten, sondern vielmehr das Organon einer noch zu vollendenden, zu bildenden, ja anzufangenden Literatur wäre. Ein Organon der Literatur, also eine Kritik, die nicht bloss erklärend und erhaltend, sondern die selbst produzierend wäre, wenigstens indirekt durch Lenkung, Anordnung, Erregung. Die Aufgabe einer solchen die Literatur in ihrem ganzen Umfange erst konstituierenden und organisierenden Kritik zerfällt in mehrere dem einen Zweck gleich dienende, aber doch wesentlich getrennte Geschäfte. ${ }^{\text {I20 }}$

Dieses gewünschte, projizierte Organon sowie dessen Zerstreuung in "wesentlich getrennte Geschäfte« verändert, so denke ich, in entscheidender Weise das übliche Verständnis von `Immanenz bzw. ıinnerer oder simmanenter Kritikı. Denn die Kritik fängt mit dem Werk als individuelles Ganzes an, ergänzt es aber kontinuierlich im Prozess der zyklischen Lektüre, der ständigen Aneignung immer neuer Formen und Kategorien und deren Kritiken. Genauer gesagt: der Kritiker beginnt mit dem Werk intensiv als einem Individuum - dessen vier zentrale Kategorien sind in Vom Wesen der Kritik Form und Gehalt, Absicht und Tendenz - bezieht es jedoch zunehmend und extensiv durch die in den Notizheften Zur Philologie herausgearbeiteten philologischen Kategorien - Verhältnisse, Arten/Gattungen, Zweck, Grenzen, Stufen, Gesetze, Lage, Ganzheit und Klassizität $t^{21}$ - auf ein System.

II8 KFSA 2, S. I4O.

II9 $K F S A$ I8, S. I25, Nr. 24.

I20 KFSA 3, S. 82.

I2I KFSA I6, S. 35, Nr. 8. Vgl. Dierkes: Literaturgeschichte als Kritik (s. Anm. 2), S. I25, hat als erster Schlegels >Kategorientafel thematisiert, ist aber nicht 
Das aber heißt: Die Kritik, so mannigfaltig sie nach Schlegel sein mag, ist systemtheoretisch gesehen ein Medium zweiter Ordnung. Im Medium der Kritik lernt sich das historische Subjekt kennen, und zwar durch Mitteilung. Ein Kunsturteil steht zunächst provisorisch `da als kritisches Factum. Im Gespräch über die Poesie aus dem Jahr I8oo heißt es dazu: „Ein wahres Kunsturteil, werden Sie mir eingestehen, eine ausgebildete, durchaus fertige Ansicht eines Werks ist immer ein kritisches Factum «. ${ }^{122}$ Aber dieses 'Factum « wird erst in der Mitteilung, und besonders in der schriftstellerischen Mitteilung, im (kritischen) Gespräch mit anderen `Eingeweihten` zu dem, was es eigentlich für die Kunst, Wissenschaft und Geschichte der Kritik bedeutet. Die von Schlegel eben dort erwähnte »Mitteilung des Kunsturteils « ${ }^{123}$ erfolgt hauptsächlich über die Medien Journal und Zeitschrift, wie etwa im Athenaeum. ${ }^{\mathrm{I} 24}$ Die kritische Lektüre ist insofern Teil des Spiels der Mitteilung gegenüber anderen, wobei Mitteilung nicht die Kommunikation eines bereits erfassten Gehalts ist, sondern, wie Schlegel deutlich macht, ein Sich-Ins-Gespräch-Einlassen, ein Treffen in und mit der Sprache vermittelst der Schrift. ${ }^{125}$ Die Kritik muss mitgeteilt werden, um ihren hermeneutischen An-

näher darauf eingegangen. Hermann Beisler: »Friedrich Schlegels kritische Kategorien«. In: Scientia Poetica 7 (2003), S. 127-I47, hier: S. I41, erläutert sehr deutlich den entscheidenden Aspekt: »das Ganze von Schlegels Kategorientafel macht einen ausgewogenen symmetrisch aufgebauten Eindruck. Schlegel stellt auch Querverbindungen her, um diese Systematisierung noch zu festigen «. Beissler versäumt aber nicht den nächsten Schritt, den systematischen Ort der Kategorien im System der Kritik zu bestimmen.

I22 KFSA 2, S. 349.

I23 Ebd..

I24 Gegen die zeitgenössische System-Theorie, genauer die durch Leibniz und Wolff in Deutschland verbreitete Metaphysik, die solche Denkeinstellungen als Teile eines funktionalen Systems verstanden hat, welches einerseits den Buchmarkt und andererseits die Leserschaft umschließt, plädiert Schlegel für ein nicht bloss funktionales System, weil das System selbst dynamischwirkend, offen und geschlossen ist, und daher keine ontologische Einheit oder Identität beanspruchen darf.

I25 Vgl. Buschmeier: Poesie und Philologie (s. Anm. 32), S. I38: „Für die Theorie des kommunikativen Anschlusses bei Schlegel kommt der Schrift, der Textualität eine besondere Bedeutung zu, ohne die sein philologischer Ansatz kaum zu verstehen ist«. 
spruch im ganzen Umfang wahrzunehmen: » Was ist Interpretazion anders als mitgetheilte hermeneutische Kritik ${ }^{126}$ heißt es im Heft Zur Philologie II.

\section{Der Systembegriff beim jungen Friedrich Schlegel}

Wie anfangs angedeutet, ist die Frage nach dem System bzw. nach der Systematik bei Schlegel in der Forschung wiederholt aufgeworfen worden. Das geschah in zweifacher Hinsicht: erstens (I) wird gefragt, ob sich Schlegels Sätze und Fragmente zu einer Theorie der Kritik und in einem kohärenten Sinn zu einem 'System`zusammenschliessen lassen, und zweitens (2) will man wissen, was eigentlich 'System bei Schlegel überhaupt bedeutet. Benjamin fasst System bei Friedrich Schlegels als etwas dem Begriff, bzw. dem Terminus, und d. h. der Sprache Innewohnendes auf. Diese strukturelle Terminologie siedelt Benjamin jedoch, wir wir anfangs zitiert haben, "jenseits von Diskursivität und Anschaulichkeit « ${ }^{127}$ an. Was Benjamin hier anzudeuten versuchte und bei den Romantikern zu entdecken glaubte, ist nicht anderes als eine völlig andere Form der Erkenntnis, die weder begrifflich-diskursiv noch rein anschauend und rezeptiv auf Empfindungen gegründet ist. Was aber eigentlich mit dem Wort `jenseits` gemeint ist, bleibt unklar. Hier zeigt sich bei Benjamin eine gewisse Tendenz zum Mystizismus, den Schlegel in den Heften Zur Philologie ausdrücklich vermeiden wollte. Wir müssen dementsprechend die Kritik und deren putatives System bei Schlegel nicht als jenseits aller Diskursivität und Anschaulichkeit, sondern eben in der kontinuierlichen Verschränkung von Begriff und Anschauung denken.

Es gibt ausgesprochen zahlreiche vielversprechende, jedoch gleichzeitig auch unbefriedigende Fragmente und Notizen zum Begriff `System` bei Schlegel. In der Lektüre seiner Äußerungen zum System stehen wir vor einer hermeneutischen Entscheidung: entweder entzieht sich der Kritikbegriff jeder systematischen Orientierung und man behauptet, Schlegels Aussagen diesbezüglich seien

I27 Benjamin: Der Begriff der Kunstkritik (s. Anm. 7), S. 47. 
inkohärent oder falsch; oder wir versuchen, diese Äußerungen in einem strukturellen, konsistenten Zusammenhang plausibel zu machen und auf diese Weise ernst zu nehmen. Die neuere Forschung neigt dazu, dem Systematischen bei Schlegel - sei es als Tendenz, als >regulativ`, oder als ıgenetische Ontologie verstanden - Rechnung zu tragen. ${ }^{128}$ Nach Dorit Messlin habe Schlegel »den Rahmen und die Grundlinien der seit den Altertumsstudien reflektierten methodischen Verfahren der Kritik und der Hermeneutik durchaus systematisch erarbeitet. «129 Auch May Mergenthaler schlägt vor, die Schlegelschen Fragmente »als Modell der vollendeten Mitteilung zu lesen ${ }^{{ }^{130}}$; nach Mergenthaler bilden sie ein »offenes System. «" ${ }^{131}$ Mergenthaler wendet sich dabei gegen Peter Fuchs, der, auf Luhmann gestützt, 1993 ein systemtheoretisches Argument zu Schlegels fragmentarischem Schreiben vorgebracht hat, demzufolge die Fragmente gänzlich in sich abgeschlossen und daher keiner weiteren Kommunikation fähig seien. ${ }^{132}$ Nach dieser Analyse seien die Fragmente nur Aussagen oder Aussprüche, die keine Erwiderung oder Auseinandersetzung mehr erlauben. Die frühromantischen Fragmente bildeten ein geschlossenes System. Luhmann selbst hat in seinem Buch Art as Social System die frühromantische Kritik einer systemtheoretischen Analyse unterzogen:

The problem became how to deal further with the excess of communicative possibilities and with the interminability (or connective uncertainty) of communication, if this problem could not be solved by the individual subject. Romanticism solved it by introducing the notion of art criticism.

I28 Vgl. neuerdings Martin Sticker/Daniel Wenz: »System und Systemkritik - Witz und Ironie als philosophische Methode beim frühen Friedrich Schlegel«. In: Philosophisches Jahrbuch I20 (2013), S. 64-8I. Die Autoren arbeiten bei Friedrich Schlegel ein »offenes System» heraus, sehen dies aber durch Witz und Ironie gesteuert und vernachlässigen dabei die systematische Funktion der Kritik.

I29 Messlin: Antike und Moderne (s. Anm. I9), S. 86.

I30 Mergenthaler: Zwischen Eros und Mitteilung. Die Frühromantik im Symposion der "Athenäums-Fragmente". Paderborn u. a. 2012, S. 22.

I3I Ebd.

I32 Peter Fuchs: „Die Form romantischer Kommunikation«. In: Athenäum 3 (1993), S. 199-222. 
The notion that criticism is an essential component in the perfection of art acknowledged for the first time the status of theory as a self-description of the system within the system (Hervorhebung von mir, RL). ${ }^{133}$

When dealing with romantic criticism, we must disregard any potential analogies to science, in particular the notion that the convergence of critical opinions indicates their truth. This notion must be sacrificed. In romanticism, individual differences between aesthetic judgments are considered normal and legitimate. There was nothing offensive about disagreement, nor did it diminish the value of critical judgment. This suggested that the objects themselves, if they mediate communication in the form of artworks, served as an equivalent for the kind of security that was accomplished verbally only via consensus or dissent - an equivalent that secured the continuation of auto-poietic communication. Criticism was already a program for an observation of the second order - for an ability to distinguish that, for its part, could be distinguished and did not need to strive toward convergence. But if this was so, communication had to allow itself to be supported by perceptible objects in order to compensate for its boundless insecurity (Hervorhebung von mir, RL). ${ }^{134}$

Halten wir fest: in Luhmanns systemtheoretischer Analyse frühromantischer Kritik fungiert das Werk als unhintergehbares Individuum, welches die unendliche Kritik als auto-poietische Kommunikation absichert. Das Ziel ist nicht Konvergenz der Meinungen (Kunsturteile) oder Kohärenz, sondern Steigerung oder Potenzierung des Dissenses, Intensivierung der Gegenstrebigkeiten ohne Ende.

Ich möchte zum Material, zu den Texten zurückkehren und Schlegels zerstreute Notizen und Aphorismen zum System, präziser zum System der Kritik einer Lektüre unterziehen, um die rauto-poietischer Charakterisierung Fuchs' und Luhmanns - „the self-description of the system within the system « - zu überprüfen. Tatsache ist, dass Schlegel selbst in einer ganzen Reihe von Aussagen auf der Notwendigkeit eines Systems und auf der Systematizität der Kritik beharrt hat. Einige davon seien hier angeführt:

I33 Niklas Luhmann: Art as Social System. Stanford 2000, S. 285.

I34 Ebd., S. 286. 
Es ist gleich tödlich für den Geist, ein System zu haben als keins zu haben. Er wird sich also entschliessen müssen, beides zu verbinden. ${ }^{135}$ Künftig ein Codex der Philologie. System.[atische] Darstellung der Grundsätze mit Hinweisung auf Anwendung. ${ }^{136}$

Die $\varkappa_{[}[$Kritik] ist nicht absolut. Ohne $\sigma \cup \sigma \tau[$ System] gelangt sie nicht zur Charakt[eristik]. ${ }^{137}$

Kritizism ist identisch mit Systematism. ${ }^{138}$

Wenn es einen Kritizismus giebt, so muß es eine ächte Methode und ein ächtes System geben, die unzertrennlich sind. - System ist eine durchgängig gegliederte Allheit von wissenschaft[lichem] Stoff, in durchgängiger Wechselwirkung $\mathrm{u}[\mathrm{nd}]$ organischem Zusammenhang. ${ }^{139}$

Alles $\sigma \cup \sigma \tau\left[\right.$ System] ist historisch und umgekehrt. ${ }^{\mathrm{I} 40}$

Historie ist kritische Wissenschaft, oder ein kritisches System, kritisiertes und kritisierendes. ${ }^{\mathrm{I}}{ }^{\mathrm{I}}$

Der systematische Gang besteht in einem Cyclus von einem theoret[ischen] und prakt[ischen] und poetischen Teil. ${ }^{\mathrm{I} 2}$

Ohne Prinzipien kein System und umgekehrt. Ein gebildeter und sich bildender Mensch ist wenigstens eine Approximation zu einem

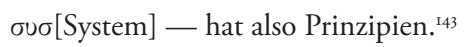

Die Philologie ist kein Aggregat von Wissenschaften, sondern ein Ganzes. ${ }^{144}$

Mit Blick auf den im Athenaeum zu findenden und berühmt gewordenen Satz - es sei gleich tödlich für den Geist, ein System zu haben als keins zu haben - stellt sich die Frage, was es bedeutet, >beides zu verbinden. Die einzig logische Möglichkeit einer Auslegung von Schlegels Fragment, vorausgesetzt, dass Schlegel Leben und Liebe dem Tod vorzieht, ist folgende: man muss viele

I35 KFSA 2, S. I73, Nr. 53.

I36 KFSA 16, S. 46, Nr. 133.

I37 KFSA I8, S. II7, Nr. 1063.

I38 Ebd., S. I25, Nr. 57.

I39 Ebd., S. I2, Nr. 84.

I40 Ebd., S. 85, Nr. 67 I.

I4I Ebd., S. 86, Nr. 676.

I42 Ebd., S. I09, Nr. 962.

I43 Ebd., S. 88, Nr. 709.

I44 KFSA I6, S. 40, Nr. 60. 

sehr schön mit Schlegels Anerkennung der immanenten Multiplizität der Kritik; denn jede Art oder Gattung der Kritik - die mikrologische, die höhere, die mystische, die philosophische, die konjekturale, die divinatorische usw. - ist auch ein System für sich, mit seiner eigenen Geschichte, seinen eigenen Gesetzen oder Spielregeln, und seinen eigenen Verfahrensweisen: jedes System bildet, so könnten wir in Anknüpfung an Sellars behaupten, eine Art `logischen Raum ${ }^{145}$ Und diese Vielfalt verträgt sich auch sehr schön mit Schlegels genetisch-ontologischem ${ }^{146}$ Trivium von Einheit, Vielheit, und Allheit, welches er der klassichen Antike entnimmt. ${ }^{\text {I47 }}$ Nun stellt sich die doppelte Frage, wie sich erstens die Einheit des Werkes zur Vielheit der Arten und der Kategorien der Kritik, und wie sich zweitens die Vielheit verschiedener kritischer Methoden und Kategorien zur Allheit oder zum System der Systeme oder zum kritischen System als solches verhält. Hier läßt sich Schlegels Systembegriff genauer präzisieren - trotz der heterogenen und oft paradoxen Formulierungen: erstens (I) jedes Individuum ist auch ein `System ` von weiteren Individuen, wie ein System selbst - als einheitliches Ganzes betrachtet - auch ein Individuum ist; zweitens (2) alle Systeme sind historisch-genetisch, und entfalten sich durch Kritik, bzw. Steigerung/Potenzierung von innen wie von

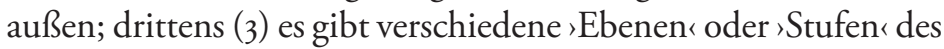
Systems; und endlich (4) viertens, jedes kritische System ist offen, und d. h. einer unendlichen, im Prinzip unabschliessbaren Kritik fähig, die selber dann entweder im System aufgenommen oder ausgestossen wird. Strukturell gliedert sich das System horizontal in einer syntagmatischen Kette wie vertikal in ein paradigmatisches Gebilde, wie das folgende Schema zeigt. ${ }^{\mathrm{I} 4^{8}}$ (Fig. I)

I45 Für den Hinweis auf Sellars danke ich Jure Zovko.

I46 Zur Konzeption der 'genetischen Ontologie bei Schlegel vgl. Messlin: Antike und Moderne (s. Anm. I9), S. 379-384.

I47 Diese Dreieinigkeit kommt sehr früh in Schlegels Denken zum Ausdruck, so etwa in Über das Studium der griechischen Poesie; vgl. KFSA I, S. 289: "das Ganze, dessen Bestandtheile Vielheit Einheit und Allheit sind."

I48 In einem zweidimensionalen, unbeweglichen Schema kann man sicherlich den Prozess der Zyklisation und Steigerung nicht darstellen; wenn man jedoch die Fortsetzung des Prozesses als ständige Rückkehr zur Materie (Werk/Sys-

\section{Gattungen und System der Kritik beim jungen Friedrich Schlegel}




\begin{tabular}{|c|c|c|}
\hline \multicolumn{3}{|c|}{$\begin{array}{c}\text { Hauptprinzip der Kritik: »Totalisation von unten herauf« }(\text { KFSA 16, S. } 68 \text { Nr. 84) } \\
\qquad \rightarrow \text { Potenzierung/Steigerung der Kritik } \rightarrow\end{array}$} \\
\hline Einheit $\rightarrow$ & $\rightarrow$ Vielheit & $\begin{array}{l}\rightarrow \text { Allheit (das Unendliche, die Fülle, } \\
\text { das Absolute) = das System aller } \\
\text { Systeme }\end{array}$ \\
\hline $\begin{array}{l}\text { Das Werk als Individuum als } \\
\text { Gegenstand der Kritik } \\
\text { Das Werk oder >Systeme von } \\
\text { Werken` } \\
\rightarrow \text { Individuelle Charakteristik } \\
\text { Lessing-Aufsätze (KFSA 3, 60) } \\
\text { Über Goethes Meister } \\
\text { Georg Forster } \\
\rightarrow \text { Das individuelle Kunsturteil } \\
\rightarrow \text { Individualität/Eigentümlichkeit } \\
\text { der Form (R. Campe) bzw. } \\
\text { »symbolische Form« (A. } \\
\text { Erlinghagen) }\end{array}$ & $\begin{array}{l}\rightarrow \text { Modi der Kritik: } \\
\text { encylopädische, mikrologische, } \\
\text { etymologische, grammatische, } \\
\text { mystische, philosophische, } \\
\text { tranzendentale, poetische, } \\
\text { nachkonstruierende, } \\
\text { konjekturale, politische, } \\
\text { hermeneutische, divinatorische, } \\
\text { philologische, Kunstkritik, } \\
\text { ästhetische Kritik, historische } \\
\text { Kritik, Textkritik (die niedere } \\
\text { Kritik) }\end{array}$ & $\begin{array}{l}\rightarrow \text { Systematische Kritik }\left(\kappa^{2}=\text { Kritik }\right. \\
\text { der Kritik wie die Verbindung } \\
\text { zwischen allen einzelnen Formen der } \\
\text { Kritik) } \\
\text { Das >neue Organon }<\text { der Literatur } \\
\text { »die noch nicht vorhandene } \\
\text { 'Wissenschaft««. }(K F S A 3,82) \\
\text { „Organismus aller Künste und } \\
\text { Wissenschaften, das Gesetz und die } \\
\text { Geschichte dieses Organismus« } \\
\text { (Lessing-Aufsatz KFSA 2, 410-411) }\end{array}$ \\
\hline $\begin{array}{l}\text { Buchstabe (fixierter Geist, Polemik) } \\
\text { und Geist (das Ganze des } \\
\text { lebendigen Zusammenhangs, } \\
\text { Kritik) in ständiger } \\
\text { Wechselwirkung: } \\
\text { »Die Lehre vom Geist und } \\
\text { Buchstaben [...][bringt] die } \\
\text { Philosophie mit der Philologie in } \\
\text { Berührung« (KFSA 2, 179, 93) } \\
\text { Das >Ganze«als »Tendenz des } \\
\text { Ganzen auf dessen Zentrum«bzw. } \\
\text { »auf einen unsichtbar bleibenden } \\
\text { Konvergenzpunkt«. (A. } \\
\text { Erlinghagen) }\end{array}$ & $\begin{array}{l}\rightarrow \text { Kritik }^{2} \\
\rightarrow \text { Kritische Kategorien bzw. }_{\text {Kategorientafel (H. Beisler): }} \\
\text { (Zur Philologie)Verhältnisse, } \\
\text { Arten/Gattungen, Zweck, } \\
\text { Grenzen, Stufen, Gesetze, Lage, } \\
\text { Ganzheit, Autor und Klassizität } \\
\rightarrow \text { Kritische Formen: } \\
\text { Kommentar, Scholien, Glossen, } \\
\text { Rezension, Lexika, Codex, } \\
\text { Charakteristik, Studie, Skizzen, } \\
\text { Fragmenten, Essays, Studie, } \\
\text { Compilation, Vorlesung usw. } \\
\rightarrow \text { Studium (KFSA 3, 53) }\end{array}$ & $\begin{array}{l}\rightarrow \varphi \sigma \varphi \lambda[\text { Philosophie der } \\
\text { Philologie] } \\
\text { Kritische/kritisierte Philosophie }=\varphi \sigma^{2} \\
\text { Philogisierte Philosophie } \\
\kappa[\text { Kritik] der } \varphi \sigma[\text { Philosophie] }]= \\
\varphi \lambda[\text { Philologie] der Philosophie. } \\
\text { (KFSA 18, S. } 40 \text { Nr. 228) } \\
\text { Diese >philologisierte Philosophie } \\
\text { müsse »kritisiende und kritisierte« } \\
\text { zugleich sein (Kritik an Kant, } \\
\text { Fichte). }\end{array}$ \\
\hline $\begin{array}{l}\text { Immanenz/innere Struktur: Form, } \\
\text { Gehalt, Absicht, Tendenz und Stil } \\
\text { des einzelnen Werks (KFSA 3, 51- } \\
60) \\
\text { Werkintention bzw. >Sinn } \text { des } \\
\text { Werkes statt Autorintention (H. } \\
\text { Dierkes) }\end{array}$ & $\begin{array}{l}\rightarrow \text { Exoterik: zyklisches } \\
\text { Lesen/zyklische Lektüre und } \\
\text { Studium } \\
\text { »Enzyklopädie« } \\
\text { „Encylopädie und Kritik sind } \\
\text { unzertrennlich verbunden« } \\
\text { (KFSA 18, S. 202, Nr. 62) }\end{array}$ & $\begin{array}{l}\rightarrow \text { Verbindung/Verflechtung von } \\
\text { Immanenz und Exoterik } \\
\rightarrow \text { Verwicklung von Theorie/Struktur } \\
\text { und Geschichte, Kunst und } \\
\text { Wissenschaft, Analytik und } \\
\text { Synthetik, Begriff und Anschauung } \\
(K F S A 13,231)\end{array}$ \\
\hline $\begin{array}{l}\text { Kunstsinn/Kunstgefühl } \\
\text { Kritik/Hermeneutik } \\
\text { Anschauung } \rightarrow » \text { Sinn fürs Ganze« } \\
\text { »Anschauung des Ganzen« } \\
\text { (KFSA } 2,413) \\
\text { Winckelmann: die >absolute } \\
\text { Verschiedenheit (M. Jäger) }\end{array}$ & $\begin{array}{l}\rightarrow \text { Kunstwissenschaft } \\
\rightarrow \text { Kritik/Hermeneutik }{ }^{2} \\
\text { =Hermeneutische Kritik: } \\
\text { >besser verstehen }<\text { und } \\
\text { >ebensogut verstehen< bzw. } \\
\text { Unverständlichkeit } \\
\text { (Grammatik/Logik) } \\
\rightarrow \text { Begriff: >Zergliederung } \\
\text { (Analyse) }\end{array}$ & $\begin{array}{l}\rightarrow \text { Wissenschaftskunst } \\
\rightarrow \text { Kritische Hermeneutik } \\
\text { »mitgeteilte hermeneutische Kritik« } \\
\rightarrow \text { Intuitives Verstehen } \\
\text { »Intuitive understanding« (E. } \\
\text { Förster) } \\
\rightarrow \text { Synthese von intuitiver } \\
\text { Anschauung und zergliedernden } \\
\text { Analyse, Diskursivität }\end{array}$ \\
\hline
\end{tabular}

Fig. 1: Friedrich Schlegels System der Kritik: ansteigende Synthesen bzw. Systematisierung durch (1) Bestätigung durch Dissens bzw. gegenseitige kritische Anerkennung, (2) Reflexion auf die (sprachlichen, schriftlichen) Formen, Gattungen und Kategorien der Kritik, und (3) ständiger Rekurs auf die materielle Basis in ihrer Gegenständlichkeit.

tem von Werken) selbst begreift, und den provisorischen Charakter jeder Kritik ständig hervorhebt - auch wenn das Kunsturteil selbst als kritisches Factum Gültigkeit besitzt - so gewinnen man damit doch einen Einblick ins System des Schlegelschen Kritikbegriffs. 
Auf der ’untersten Ebene setzt die Kritik immer ein Kunstgefühl bzw. einen Kunstsinn voraus, geht immer von der strukturellen Form und vom Gehalt des einzelnen Werkes aus, und ist auf ein Kunsturteil gerichtet. Sie verfährt also zunächst immanent in dem im Schema skizzierten Sinne eines vorläufigen Kunsturteils. Ein vorläufiges Kunsturteil ist die Bedingung der Kritik dafür, dass das Werk 'Klassizität beansprucht und von daher einer im emphatischen Sinne echten Kritik überhaupt fähig ist. Die vier schon erwähnten zentralen Kategorien in Vom Wesen der Kritik - Form und Gehalt, Absicht und Tendenz - werden auf dieser Ebene am einzelnen Werk wie im System der Werke (des Verfassers, der Epoche, der Gattung und des Stils) untersucht. Aus dieser Ebene der Kritik entsteht die individuelle Charakteristik eines Werkes.

Solche Kritik mündet in die für Schlegel in diesem Zusammenhang vorbildliche Form der Charakteristik, welche die Eigentümlichkeit, den `Geist` und den `Sinn` des Werks zu fassen sucht. Auf dieser Ebene der Kritik bedarf es hauptsächlich eines Sinns fürs Ganze und der Anschauung - wieder im oben genannten Sinn von Anschauung als Ahnung und Erinnerung - der innewohnenden Einheitlichkeit und Individualität des Werks, die Armin Erlinghagen als »Gesetzmäßigkeit des Gegenstands" als `Ganzes` bezeichnet hat. ${ }^{149}$ Dieses Werk und seine Kritik werden dann einer Reflexion unterzogen, die auf eine höhere, d. i. mehr reflektierende zweite Ebene der Kritik deutet, wo sowohl die Kategorien der Kritik wie die schriftlichen Formen der Kritik am Werk thematisiert werden. Zugleich wird das Werk in ein System oder in eine Struktur eingeordnet (so wird z. B. Goethes Meister als Quintessenz der Moderne verstanden), wobei seine eigentümlichen Eigenschaften und Tendenzen in Bezug auf ein umfassendes System von Werken (z. B. ımoderne` oder `künstlicher Bildung), gesetzt werden. Aufgrund der von Winckelmann übernommenen sabsoluten Verschiedenheit‘ sollten dann weiter die Aspekte des Systems selbst kritisiert und d. h. in der Mitteilung kommuniziert werden, wobei der ganze hermeneutische Apparat selbst einer kritischen Reflexion ausgesetzt wird. Erst auf dieser

I49 Erlinghagen: "Das Konzept des 'Ganzen` in Friedrich Schlegels Poetik" (Anm. Io), S. I8. 
140 zweiten Ebene spielt sich das von Schlegel geforderte sbesser verstehen` bzw. ^ebensogut verstehen` ab: nur hier wird die Konjekturalkritik wie die niedere Textkritik transzendiert und auf das Interpretatorische konzentriert, so dass die Art und das Verfahren der Kritik selbst in den Vordergrund rückt. Dieses Ganze - die einzelne Kritik und die Kritik ihrer Bedingungen - muss dann letztlich um einen weiteren Schritt erweitert werden, und zwar im Sinne einer Philosophie der Philologie als Philologie in der zweiten Potenz, die zugleich eine Philologie oder philologische Kritik der Philosophie ist. Individuelle `Kritiken ‘ beziehen sich stets auf die unendliche Verschränkung und produktive Auseinandersetzung von Struktur/Theorie und Geschichte, von Kunst und Wissenschaft, Anschauung und Begriff, Esoterik und Exoterik, Analytik und Synthetik, Kritik und Hermeneutik. Auf der Ebene des Systems (Allheit) findet eine weitere Reflexion über die genaue Beziehung zwischen dem einzelnen Werk und den kritischen Formen/Kategorien, sowie über den hermeneutischen Apparat und seine `Anwendung selbst statt. Hier lässt sich erstmals das »neue Organon" erblicken, das Schlegel in seinem Lessing-Aufsatz als ssystematische Kritikı anvisiert und als eine "noch nicht vorhandene Wissenschaft «, als "Organismus aller Künste und Wissenschaften, das Gesetz und die Geschichte dieses Organismus « vorstellt. Auf dieser dritten Stufe durchdringen sich intuitive Anschauung und diskursive Begrifflichkeit, analytisches Verfahren und synthetische Bildung, Immanenz und Exoterik, intensives 'Studium ' und rzyklisches Lesen`, philologische Forschung und philosophische Totalität/Universalität, aber mit dem Vorbehalt, dass dieses 'Ganzers stets auf die philologische Materie selbst, auf die Texte selber, wie auf die Kategorien und Formen rekurriert. Weder als Bild noch als Schema zu fassen, muss Schlegels System der Kritik als selbstkritischer Prozess und als rekursives Verfahren gedacht werden, nach welchem man sich kontinuierlich auf die materielle philologische Basis besinnt, aufsteigende Synthesen erstrebt, und, wenn nötig, etwas am System ändert. Die doppelte Bewegung des kritischen Systems, zugleich kritisiertes und kritisierendes zu sein, wie Schlegel sich ausdrückt, steht als Garant dafür, dass das System selbst als Ganzes nie als etwas Gegebenes substanzialisiert 
werden kann. ${ }^{150}$ Als offenes, immer im Werden begriffenes System fängt es immer wieder von unten an. Es bestätigt sich durch Rekurs auf das einzelne Werk oder das System der Werke, auf gegenseitige Anerkennung durch Kritik und im Gespräch mit anderen Ansichten und Meinungen.

Wir sind aufgrund des hier skizzierten Argumentes im Stande, Schlegels Systembegriff von Luhmanns und Fuchs' systemtheoretischer Charakterisierung der frühromantischen Kritik abzugrenzen. ${ }^{\mathrm{ISI}}$

I50 Hier stimme ich mit Erlinghagen: „Das Konzept des `Ganzen` in Friedrich Schlegels Poetik« (s. Anm. Io), S. I8, überein, der zum Formbegriff festhält: »Form` wird hierbei nicht objektivierend oder substanzialisierend begriffen [...] es handelt sich vielmehr um einen operativen Begriff, vermittels dessen das Vorgestellte sowohl statisiert als auch dynamisiert werden kann, so dass es als Einheit einer Vielheit ko-existenter oder sukzessiver Teile zu Gesicht kommen kann."

I5I Gleichzeitig wäre auf das poetologische Konzept des `Ganzen ` hinzuweisen, das ein wichtiges, ganz bestimmtes Moment im System beansprucht. Für Erlinghagen: »Das Konzept des `Ganzen« in Friedrich Schlegels Poetik« (s. Anm. Io), S. 6I, ist das Ganze der Zentralgedanke der Philosophie Friedrich Schlegels. Dagegen ist einzuwenden, dass sich das 'Ganze`, die symbolische Form eines Werks oder eines Euvres, letzten Endes nur im Rahmen der Allheit des Gesamtsystems verstehen ließe, welches Schlegel als zukünftiges Organon aller Wissenschaft begreift. Jedoch beruht alles auf dem Unterschied zwischen dem Ganzen als Totalität im Sinne der Gesamtheit, der Allheit, und dem Ganzen als poetologisches Kriterium, als Formprinzip, als Einheit. Erlinghagen erklärt nur letzteres: " Einheit ist der Name für die Form des Ganzen. «(Ebd., S. 48) Hier liegt auch ein Gegenargument zur These, das Ganze sei Bei Schlegel das »alles und jedes durchdringende Konzept «(ebd., S. 6I). Das Konzept des `Ganzen ist - zumindest in der Moderne - immer wieder mit Kant, und d. h. mit einem transzendentalphilosophischen Ansatz verbündet. Dies ist auch bei Erlinghagen der Fall: »Um als ein Ganzes erscheinen zu können, muss die Konstituierung eines jeden Ganzen bestimmten Regeln folgen, deren Struktur - ein Spezifikum von Schlegels Philosophie - fundamental der transzendentalen Verfassung des menschlichen Gemütsvermögen, im Sinne der kritischen Philosophie, korrespondiert. (Ebd., S. 40 f.) Es stellt sich aber die Frage, inwiefern man noch ein transzendentales Argument - das 'Ganzer sei die Einheit der Form, ein Konvergenzpunkt verschiedener Anschauungen - angesichts von Schlegels scharfer Kritik an Kant und an dessen transzendentalphilosophischem Ansatz ohne Bedenken aufstellen kann. Erlinghagens Theorie des ‘Ganzen bei Schlegel als »ontologisches und epistemologisches Modell« (ebd., S. 22) scheint mir tatsächlich Schlegels kritisches System auf einen literaturkritischen bzw. poetologischen Mittelpunkt zu reduzieren.

\section{Gattungen und System der Kritik beim jungen Friedrich Schlegel}


142 Die Aporien dieses Systems bestehen nach Ansicht der Systemtheorie einerseits in einem Überschuss kommunikativer Möglichkeiten, der prinzipiellen Unabschliessbarkeit der Auslegung und der ıunsicheren`Konnektivität der Elemente, schliesslich in der Verzerrung, der ein solches Systems wegen der ständigen Mitteilung ausgesetzt ist; andererseits sichert es sich als autopoietisches System esoterisch von weiteren (bedrohlichen oder für das System gefährlichen) Kommunikationen ab. Da im frühromantischen System der Kritik kein Rekurs mehr auf das Subjekt (vgl. Schlegels Kritik an Kants Kritik der Urteilskraft), noch auf eine Konvergenz der Meinungen - vielmehr ist der Dissens erwünscht! - möglich ist, fungiere der Gegenstand selbst (»the object itself ", »the perceptible object« - nämlich `das Werk» nach Luhmann) als Ausgleich, als Kompensation für diese starke begriffliche Instabilität ${ }^{52}$. Während der erste Teil dieses Arguments eine beschränkte Gültigkeit hat (in Bezug auf den Überschuss kommunikativer Möglichkeiten und die grundsäztliche Unabschliessbarkeit der Kritik), hat es sich in unserer Analyse doch gezeigt, dass sich das frühromantische System der Kritik Schlegels nur als ein ıoffenes System im emphatischen Sinne auffassen läßt; ${ }^{\mathrm{IS} 3}$ und dass der `Gegenstand ( (das Werk oder ein System von Werken) zwar den Anfang, aber bei weitem nicht das Ganze des kritischen Prozesses darstellt. Luhmanns Systembegriff und insbesondere sein Konzept des autopoietischen Systems mit seiner ’operativen Geschlossenheit`steht im starken Gegensatz (I) zu Schlegels reflektierender Steigerung/Potenzierung des Werks selbst vermittelst der Kritik, das sich (als System selber) der Kritik öffnet; (2) zu Schlegels Mitteilung und Mitteilbarkeit der Kritik, die ihrerseits dann weitere Verwandlungen im System

I52 Der kursiv gesetzte Ausdruck ist eine bewusste Anspielung auf Robert Brandoms `Strong Conceptual Instability` (SCI); Robert Brandom: A Spirit of Trust. A Semantic Reading of Hegel's Phenomenology of Spirit. http://www. pitt.edu/_brandom/spirit_of_trust_20I4.html (I4.9.20I4). Die inferentielle Anschliessbarkeit von Aussagen sowie das zentrale Kriterium der Anerkennung von Anderen bilden zusammen die unausgesprochene Grundlage von Schlegels System.

I53 Auch Mergenthaler: Zwischen Eros und Mitteilung (s. Anm. I30) kommt zu dem Ergebnis, dass Schlegel ein ıoffenes` System entwirft, auch wenn ihr Akzent eher auf der ,vollendeten Mitteilung، liegt. 
herbeiführt; (3) zu Schlegels Entfunktionalisierung des Systems, insofern als die sgetrennten Geschäfter der Kritik ihrerseits einer Kritik unterzogen werden. Da es sich dabei weder um eine rregulative Idee im kantischen Sinne, noch um eine rein spekulative, völlig reflektierte, zu-sich-selbst gekommene Idee im Sinne Hegels handelt, konstitutiert sich Schlegels 'System` nur fragmentarisch im unendlichen Diskursus, im ständigen hin und her zwischen der materiellen Textbasis (dem Werk), den verschiedenen Gattungen und Modi der Kritik und deren Kategorien, zwischen hermeneutisch-kritischer Reflexion und den immer höheren Synthesen, die sich aus dem hermeneutischen Prozess herauskristallisieren. Dieser Prozess unterliegt dem Imperativ der Mitteilung und der Anerkennung einer sich erst bildenden Öffentlichkeit. Das neue Organon, das Schlegel im Lessing-Aufsatz kundtut, ist nichts anders als dieses aus der Vielfalt der Kritik heraus sich realisierende System der Kritik selbst. Es ist ein historisches, offenes und sich verwandelndes System. Im Gegensatz zu Luhmann ist aber die Kritik beim jungen Friedrich Schlegel keines Ausgleichs und keiner Kompensation bedürftig: Unsicherheit und Instabilität sind notwendige Konsequenzen der Kritik selber, die immer zurück zur Materie, also zu den sprachlich-schriftlich konstitutierten Sätzen und Werken zurückgeht, und von unten herauf immer wieder - freilich mit neu gewonnenen Erkentnnisssen und mehr Kunstsinn - den kritischen Prozess befeuert.

\section{Fazit}

Der Beitrag hat die Frage aufgeworfen, ob Schlegels mannigfaltige Äußerungen zur Kritik und zum System derselben wirklich ernst zu nehmen sind. Auch wenn dieses System nie eine ausführliche, explizite Darstellung bei Schlegel gefunden hat - in seinen Vorlesungen kommen wir einer solchen Darstellung des Systems am nächsten - ist die Evidenz des Entwurfs eines kritischen Systems bei Schlegel überwältigend. Die Kritik wird weder durch individuelle Charakteristiken und einzelne Analysen bzw. kritische Kunsturteile, noch durch die Vielfalt der Formen und Kategorien der Kritik, noch letztendlich durch eine kritische Reflexion der Kritik selbst - durch 
eine Kritik der Kritik (im Sinne Frischmanns) - erschöpft. Vielmehr hat sich ergeben, dass die Vielfalt von kritischen Formen, Verfahrensweisen und Kategorien stets auf ein System bezogen ist, welches sich genetisch-geschichtlich als ein sich bildendes Organon konstitutiert. ${ }^{\mathrm{IS4}}$ Ohne systematischen Impuls bleibt die Kritik der Willkür, dem Belieben und dem Geschmack des Einzelnen oder einer jeden Kultur preisgegeben, was freilich dem Projekt der Kritik im Sinne Schlegels grundsätzlich widerspricht.

Es stand hier aber noch mehr auf dem Spiel. Seit Jahren schon ist der Begiff des Systems in den Geisteswissenschaften wie in der europäischen Philosophie weitgehend verpönt. Er wird als begriffliches Analogon des politischen Schreckens gedacht und mit einem rationalistischen Universalismus verbunden, der im Verbund mit Macht und Herrschaft den Ausschluss bzw. die Tilgung des Einzelnen und des Individuellen herbeiführe. ${ }^{55}$ Schlegels System der Kritik zeigt aber, dass das System nur als fliessendes, genetischhistorisches, durch gegenseitige Anerkennung und Bestätigung zu konstituierendes Gebilde besteht und als solches immer im Werden begriffen ist, weder als regulative Idee im Kantischen Sinne noch als das alles andere durch den Begriff an sich reißende Absolute im Sinne Hegels, sondern als wirklich existierendes Ganzes, welches die Kritik seiner eignen Form in sich selbst immer neu verwirklicht. Rüdiger Campe hat neulich genau dies im Hinblick auf den Begriff der Form bei Schlegel als Wendepunkt in der Geschichte der Literatur-Wissenschaft aufgezeigt:

Die Form, die das Argument von Schlegels Umgründung der LiteraturWissenschaft ausmacht, ist eine Form, die sich an ihre eigene Kritik macht. Sie ist die Kritik dessen, was sie einmal als metaphysische Form, als Idee, gewesen ist. Die Form, die sich an ihre eigene Kritik macht, wird dadurch aber auch zu einem äußerst flexiblen Instrument der Organisation. Schlegels Literatur-Wissenschaft ist nur der erste Großversuch dieser Art, die erste Anwendung flexibler Formprozesse

I54 Friedrich Schlegel: Lessings Gedanken und Meinungen (I804). In: KFSA 3 , S. 82, wo er von einem »Organon der Literatur« spricht.

I55 Vgl. vor allem Jean-François Lyotard: The Post Modern Condition: A Report on Knowledge. Minneapolis I979. 
auf einen Gegenstandsbereich des Wissens und ein Versuch, der sicher

für lange Zeit eine Modellfunktion behalten hat. ${ }^{156}$

Als Medium zweiter Ordnung muss sich die Kritik gegen jede totalisierende Funktionalisierung, gegen die Herrschaft jeder absoluten Systematisierung ihrer selbst, wehren, will sie die beiden Kriterien des (I) reflektierenden Rekursus auf den Text und die hermeneutisch-kritischen Kategorien und (2) die Gegenstrebigkeit, Anschliessbarkeit und schliesslich Anerkennung kritischer Urteile in der Mitteilung aufrechterhalten. Insofern läßt sich Schlegels Systembegriff gegenüber Hegel und der Systemtheorie Luhmanns als Alternative offerieren. Die prinzipielle Offenheit der Schlegelschen Philosophie ist gegenüber der an den abstrakten Begriff gebundenen Systemform Hegels ebenso hervorzuheben wie gegenüber Luhmanns Funktionalismus. Im Gegensatz zu Hegel und Luhmann argumentiert Schlegel für die Pluralität philosophischer Sprachen, für die Vielzahl literarisch/künstlerischer und begrifflicher Darstellungsformen, für die Mannigfaltigkeit der experimentell eingesetzten Verfahrensweisen und schliesslich auch der synthetischen Systematisierungsversuche. ${ }^{157}$ Nur durch den permanenten Rekurs auf die `Materie - hier verstanden nicht als fixiertes, unwandelbares, kompensatorisches Bestehendes, sondern gerade als wandelbare, interpretierte und zu interpretierende Gegenständlichkeit- und die kritisch-historische Revidierung ihrer eigenen Voraussetzungen und Synthesen (von Schlegel mit der Parole »Totalisation von unten herauf ${ }^{158}$ indiziert) - behält die Kritik als ständige Aufgabe ihre unvoraussehbare, kontingente Gegenmacht.

I56 Rüdiger Campe: „Das Argument der Form in Schlegels `Gespräch über die Poesier. Eine Wende im Wissen der Literatur«. In: Merkur 68 (20I4), S. IIO-I22, hier: S. I20. Campe erkennt bei Schlegel die erste explizite »Überführung der Rhetorik und der Poetik, der Literatur als figura, in die Untersuchung und das Denken der Form«: »Mit einem Schlag sind die Feinheiten und Kleinlichkeiten der rhetorischen Unterscheidungen und der poetologischen Lehren fortgewischt, und der Blick öffnet sich auf die Bahn aufsteigender Verallgemeinerungen, die im Medium der Formfrage angelegt sind« (ebd., S. I2I).

I57 Ich bin Guido Naschert für seine sorgfältige Lektüre und Kritik meines Arguments zum Systembegriff sehr dankbar.

I58 KFSA I6, S. 68, Nr. 84. 